\title{
Second-order Godunov-type scheme for reactive flow calculations on moving meshes
}

\section{REPORT}

\author{
Boris N. Azarenok ${ }^{\mathrm{a}}$, Tao Tang ${ }^{\mathrm{b}}$ \\ ${ }^{a}$ Dorodnicyn Computing Center of the Russian Academy of Sciences, Vavilov str. \\ 40, GSP-1, Moscow, 119991, Russia \\ ${ }^{\mathrm{b}}$ Department of Mathematics, The Hong Kong Baptist University, Kowloon Tong, \\ Hong Kong
}

\begin{abstract}
The method of calculating the system of gas dynamics equations coupled with the chemical reaction equation is considered. The flow parameters are updated in whole without splitting the system into a hydrodynamical part and an ODE part. The numerical algorithm is based on the Godunov's scheme on deforming meshes with some modification to increase the scheme-order in time and space. The variational approach is applied to generate the moving adaptive mesh. At every time step the functional of smoothness, written on the graph of the control function, is minimized. The grid-lines are condensed in the vicinity of the main solution singularities, e.g. precursor shock, fire zones, intensive transverse shocks, and slip lines, which allows resolving a fine structure of the reaction domain. The numerical examples relating to the Chapman-Jouguet detonation and unstable overdriven detonation are considered in both one and two space dimensions.
\end{abstract}

Key words: Detonation wave, Second-order Godunov-type scheme, Moving mesh

\section{Introduction}

Modeling detonation wave motion in gases has started in 1940s, see, e.g., [58,26], based on the theory of the steady one-dimensional detonation, referred to as the Zeldovich-NeumanDoering (ZND) model. The early computations were rather rough giving only a qualitative

Email addresses: azarenok@ccas.ru (Boris N. Azarenok), ttang@math.hkbu.edu.hk (Tao Tang). 
estimate to the solution. The main difficulty of the numerical simulation is due to the different scales of the flow domain and chemical reaction zone. Thus, the simulation for the real objects requires using more powerful computers or more sophisticated numerical algorithms.

Developing the numerical algorithms is executed in several ways. In the first group the burning zone is not resolved by the grid points. Instead in [16] the chemical heat release is put into the Riemann problem. This idea is used in [28] as well. In [15], the chemical reaction term is present only to the energy equation, the kinetics equation is omitted and the Riemann problem is formulated for the non-reactive gas. Although the detonation wave speed is obtained rather inaccurately, the calculations of the real industrial objects with complex geometry are found satisfactory. In the second group of the algorithms, the burning zone is resolved by putting there several grid points in the normal direction. This requires to use very fine quasiuniform meshes. In the most of these algorithms one applies the fractional step approach (also referred to as the Strang splitting schemes). At each time step, first, the system of conservation laws is treated and then the ODE to the kinetics equation is solved, e.g. see $[8,17,29,43,44,53]$. Although the convergence of the fractional step method was justified theoretically for scalar conservation laws with source terms $[19,52,51]$, the application for this approach for hyperbolic system with stiff source terms generally produces the nonphysical solution, e.g. see [17]. Another way is to treat the system of conservation laws coupled with the reactive equation as a whole, i.e. using the unsplit schemes. In this approach the heat release term in the right part of the system is treated as a source term. In [7], the generalized Riemann problem is introduced for the reactive equations to provide the second order approximation in time. In [20], the detonation process is simulated on the Lagrangian mesh. Space-time paths are introduced in [42] on which the equations are reduced to the canonical form about the "new" Riemann invariants. All the above methods are of Godunov-type (except [16], where the random choice method is used), i.e. include solution of the Riemann problem that allows obtaining the narrow wave front rather precisely. In contrast to it, the random projection method is used in [6] where the Riemann problem is omitted from the consideration. However, justification of such a simplification is still under the question. Some other non-Godunovtype algorithms can be found in [41].

In this work, we present an unsplit scheme for calculating the reactive flow equations on the moving meshes. For this we utilize the idea of the Godunov's scheme on the deforming meshes (see the monographs $[1,31]$ ), when the conservation laws are written in the integral form using the so-called generalized formulation in $\mathbb{R}^{3}$ space $(x, y, t)$ ( $t$ is time). This allows updating the flow parameters directly on the moving curvilinear mesh without using interpolation. One implementation of the first-order Godunov's method on the moving mesh with front tracking was performed in [27].

In [2], a modification of the Godunov's scheme of the second-order accuracy in time and space was suggested, because the first-order original scheme in [31] does not provide 
proper grid-nodes adaptation to the solution singularities. The second order in space is achieved by interpolating the flow parameters inside the cells, and in time by using the Runge-Kutta method with a predictor-corrector procedure. To obtain the fluxes value at the cell faces the Riemann problem is solved. The kinetics equation is treated similarly, namely we write it in the integral form and approximate it in the hexahedral cell in space $(x, y, t)$.

The variational approach is employed to generate the moving mesh. The variational approach to generate two-dimensional meshes was suggested in the form of quasi-conformal mapping in [30]. In [55], the variational principles for constructing the adaptive moving grids in the gas dynamics problems were formulated. They introduced the measure (or functional) of mesh deviation from the Lagrange coordinates, measure of mesh deformation and mesh concentration. In [10], the functional of smoothness was applied, to which the Euler-Lagrange equations coincide with the system used in [57]. In [39], the problem of minimizing the functional of smoothness (also referred to as the harmonic or Dirichlet's functional) written for a surface of the control/monitor function was formulated to construct an adaptive-harmonic mesh. Other forms of the monitor functions for the harmonic functional have been considered in $[21,11,50]$. In continuous approach the harmonic mapping, subject to some known conditions, is a homeomorphism. However, its discrete realization, based on solving the Euler-Lagrange equations, suffers from mesh tangling in the domains with complex geometry (e.g. nonconvex domains with singularities on the boundary). To provide an one-to-one harmonic mapping at the discrete level, it has been suggested in [13] to use a variational barrier method, which constructs the mapping by minimizing the harmonic functional. The functional is approximated in such a manner that there is an infinite barrier ensuring all grid cells to be convex quadrilaterals. In [35], the barrier method is also used together with some geometric constrains, since in the corners of the cell that barrier disappears. The approach from [13] has been extended to the adaptive grid generation in [14]. In $[2,3,4,5]$, this approach has been applied to the two-dimensional problems of gas dynamics in nonconvex domains, where the control function is one of the flow parameters or superposition of several parameters. In [4], the algorithm of redistributing the boundary nodes at adaptation, consisting in constrained minimization of the functional, was suggested. In the present work we demonstrate that by condensing the grid lines we can better resolve main singularities in the vicinity of the detonation wave, such as a precursor shock, fire zones, intensive transverse shocks and slip lines.

This article has the following structure: in section 2 we will consider several aspects in the one-dimensional case, including governing equations, boundary conditions, numerical scheme, the Riemann problem on the moving mesh, and stability condition; the twodimensional case will be considered in section 3; in section 4, the grid generation method will be presented; and in section 5 , several detailed numerical simulations will be reported. 


\section{One-dimensional case}

\subsection{System of equations}

The system of the conservation laws governing the gas flow in Euler approach and coupled with the irreversible chemical reaction equation reads

$$
\frac{\partial \boldsymbol{\sigma}}{\partial t}+\frac{\partial \boldsymbol{a}}{\partial x}=\boldsymbol{c}
$$

where $\boldsymbol{\sigma}=(\rho, \rho u, E, \rho Z)^{\top}, \quad \boldsymbol{a}=\left(\rho u, \rho u^{2}+p, u(E+p), \rho u Z\right)^{\top}$, and $\boldsymbol{c}=(0,0,0,-\rho Z K(T))^{\top}$. Here $u, p, \rho, E, T, Z$ are, respectively, the velocity, pressure, density, total energy, temperature, and mass fraction of the unburnt gas. The total energy is $E=\rho\left(e+0.5 u^{2}\right)+q_{o} \rho Z$, where $e$ and $q_{o}$ are the specific internal energy and heat release, respectively. The equation of state is $p=(\gamma-1) e \rho$, where $\gamma$, the ratio of the specific heats, is assumed to be the same in the burnt and unburnt gases. The temperature is $T=p / \rho R$, where $R$ is the specific gas constant. In order that the Cauchy problem to the system (1) without chemical reaction (added with the law of increase of the entropy $s$ across a shock) has an unique solution, the internal energy as a function $e=e(v, s)$ must be convex with respect to its arguments, i.e. the specific volume $v=1 / \rho$ and entropy $s$. More precisely,

$$
e_{v v}>0, \quad e_{v v} e_{s s}-e_{v s}^{2}>0
$$

Moreover, $e(v, s)$ should satisfy the additional restrictions, suggested in [56],

$$
e_{v s}<0, \quad e_{v v v}<0
$$

Note that till now a strict mathematical justification for uniqueness of the Cauchy problem has not been given (only in a linearized approach, see, e.g., [32]). On the other hand the statement of uniqueness has been confirmed by over 50 years numerical practice.

The internal energy $e$ of the ideal gas satisfies the conditions (2) and (3). The function $K$, referred to as the reaction rate, depends on the temperature $T$ via the Arrhenius kinetics

$$
K(T)=K_{o} \exp \left(-E^{+} / T\right)
$$

where $E^{+}$is the activation energy, and $K_{o}$ is the rate constant. Sometimes this reaction rate is replaced by a discrete ignition temperature kinetics model

$$
K(T)= \begin{cases}0 & \text { if } T<T_{\mathrm{ign}} \\ 1 / \tau_{o} & \text { otherwise }\end{cases}
$$


where $T_{\text {ign }}$ is the ignition temperature, $\tau_{o}$ is the time scale of the chemical reaction.

For the steady ZND model, we can integrate the first three ODEs in (1) and obtain the algebraic equations connecting the values ahead and behind the Chapman-Jouguet (CJ) detonation wave moving with the velocity $D_{\mathrm{CJ}}[58,26]$. As a result, the reactive equation becomes the ODE for the mass fraction $Z$. The resulting ODE can be integrated numerically, which yields an exact solution useful for the test modeling. This model is also used in the case of the overdriven (also referred to as overcompressed [58]) detonation wave.

To construct the numerical scheme on the moving mesh we will use the system (1) written in the integral form. Integrating (1) over an arbitrary domain $\Omega$ in the $x-t$ plane gives

$$
\iint_{\Omega}\left(\frac{\partial \boldsymbol{\sigma}}{\partial t}+\frac{\partial \boldsymbol{a}}{\partial x}\right) \mathrm{d} x \mathrm{~d} t=\iint_{\Omega} \boldsymbol{c} \mathrm{d} x \mathrm{~d} t
$$

By virtue of the Green's theorem we get

$$
\oint_{\partial \Omega} \boldsymbol{\sigma} \mathrm{d} x-\boldsymbol{a} \mathrm{d} t=\iint_{\Omega} \boldsymbol{c} \mathrm{d} x \mathrm{~d} t
$$

where the line integration is performed along the boundary $\partial \Omega$ of the domain $\Omega$ in an anticlock-wise manner.

\subsection{Boundary conditions}

Assume the detonation wave moves from the left to the right. Then ahead the wave there is the unburnt gas and behind (strictly speaking at the infinite distance) the completely burnt gas. Consider the matter that under what boundary conditions ahead and behind the detonation wave then the IBVP is well posed. To understand it, we analyze the linearized system of the gas dynamics equations written in the differential form as it is usually performed, see, e.g., [31],

$$
\frac{\partial}{\partial t}\left(\begin{array}{c}
\rho \\
u \\
p
\end{array}\right)+\left(\begin{array}{ccc}
u_{o} & \rho_{o} & 0 \\
0 & u_{o} & 1 / \rho_{o} \\
0 & \rho_{o} c_{o}^{2} & u_{o}
\end{array}\right) \frac{\partial}{\partial x}\left(\begin{array}{c}
\rho \\
u \\
p
\end{array}\right)=0
$$


where $\rho_{o}, u_{o}, p_{o}$ are the constant values, $\rho, u, p$ are small perturbations, and $c_{o}$ is the sound speed. This system in a standard manner can be reduced to the canonical form

$$
\frac{\partial \boldsymbol{e}}{\partial t}+\mathcal{L} \frac{\partial \boldsymbol{e}}{\partial x}=0
$$

where $\mathcal{L}$ is a diagonal matrix with the diagonal elements $\lambda_{-}=u_{o}-c_{o}, \lambda_{o}=u_{o}$, and $\lambda_{+}=u_{o}+c_{o}$. The components of the vector $\boldsymbol{e}=\left(p-\rho_{o} c_{o} u, c_{o}^{2} \rho-p, p+\rho_{o} c_{o} u\right)^{\top}$ are the Riemann invariants being constant along three characteristics $\mathrm{d} x / \mathrm{d} t=\lambda_{-}, \lambda_{o}, \lambda_{+}$, respectively.

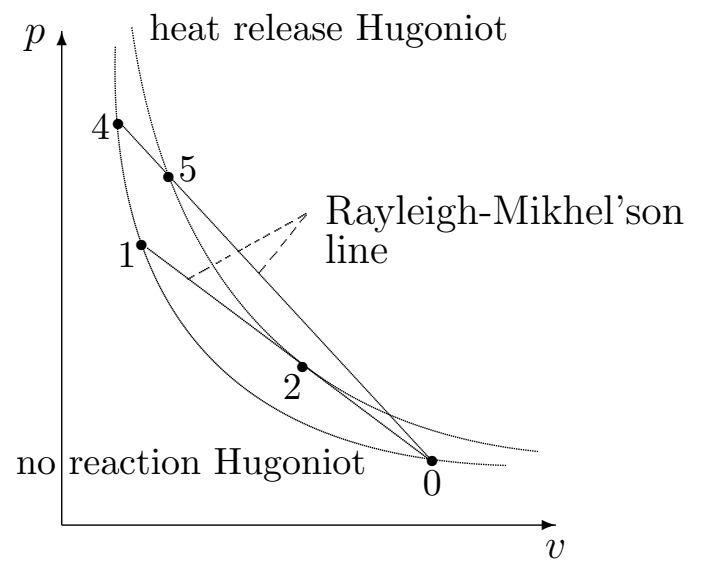

Figure 1. On the $(p, v)$ diagram, where $v$ is the specific volume, the process passing the points 0,1,2 on the Rayleigh-Mikhel'son line corresponds to the CJ solution; and the process passing points $0,4,5$ is an overcompressed/overdriven detonation.

Now consider the detonation wave in the CJ solution. It moves with the supersonic speed relative to the unburnt gas ahead the wave. For the burnt gas, behind the wave, it moves with the sonic speed. It can be seen from Fig. 1, that the Rayleigh-Mikhel'son line is tangent to the Hugoniot curve for the burnt gas. Note that the tangent line inclination to the Hugoniot curve is the isentropic derivative $(\partial p / \partial \rho)_{S}$ at point 2, i.e., the local sound speed squared in the burnt gas (for details of physics see $[58,26]$ ). In other words, the detonation wave path is space-like with respect to the regions ahead and behind it [18]. Thus, the three characteristics impinge on the wave from the unburnt gas and we should define the values of all three invariants ahead the detonation wave, i.e., the values of $u, p, \rho$. From the burnt gas no characteristic impinges the wave. Thus, no boundary conditions behind the detonation wave, say on the piston, should be specified. In practical computations we set the transmissive boundary conditions there.

Let us analyze the overcompressed detonation wave flow. This process can also be steady subject to supporting by the corresponding piston motion [58]. Here the wave front moves with the supersonic speed relative to the unburnt gas and with the subsonic speed for the burnt gas. This fact is reflected in Fig. 1, where the Rayleigh-Mikhel'son line inclination is less than the tangent line inclination at point 5 , while for the weak shock the isentrope coincides approximately with the Hugoniot curve. From the burnt gas the characteristic $\lambda_{+}$arrives the wave, transferring the Riemann invariant value $\rho_{o} c_{o} u+p$. Thus, we need 
to define its value, namely $u$ and $p$ on the rear boundary. In practice, one can define only the value of $u$, the piston velocity. The value of $p$ in the left endcell will be updated automatically from the finite difference relation approximating the third equation of (8). Generally speaking, on the rear boundary we can define the value of $p$. Then the velocity $u$ in the left endcell is updated automatically. Another question is that we do not know how to define the pressure in the left end. We may set the value of $p$ equal the atmospheric pressure if it is an open end of the tube, but the overdriven detonation can not occur in a free mode without support. On the front boundary again we should define three conditions, i.e., the values of $u, p, \rho$. For the fourth reactive equation of the system (1) the analysis is quite simple. The velocity $u$ is the characteristic (in the linearized analysis it is the constant value $u_{o}$ ). It can be treated by analogy to the second equation of $(8)$. Therefore, we only need to define the value of $Z$ for the unburnt gas but not for the burnt gas (simply one uses the transmissive boundary condition). We will return to the discussion on the rear boundary conditions at the end of this section after presenting the numerical scheme.

\subsection{Numerical scheme}

In the $x$ - $t$ plane we introduce the moving mesh, see Fig. 2 , with spacings at the $n$th time level $h_{i+1 / 2}^{n}=x_{i+1}^{n}-x_{i}^{n}$ and at the $n+1$ th level $h_{i+1 / 2}^{n+1}=x_{i+1}^{n+1}-x_{i}^{n+1}$, and time step $\Delta t=t^{n+1}-t^{n}$, where $n, i$ are positive integers. Furthermore, for conciseness whenever possible, we will omit the superscript and subscript for the time index and use convention that, for instance to the spacing $h$, using the subscript $h_{i+1 / 2}$ denotes $h_{i+1 / 2}^{n}$, the value at the bottom time level $n$; and superscript $h^{i+1 / 2}$ denotes $h_{i+1 / 2}^{n+1}$, the value at the top time level $n+1$.

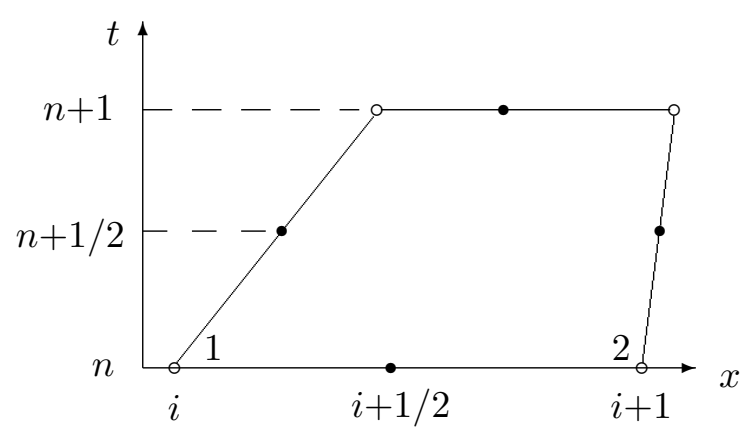

Figure 2. A quadrilateral (trapezoidal) cell $\Omega_{i+1 / 2}^{n+1 / 2}$ in the $x$ - $t$ plane, which corresponds to two states of the control volume $\left(x_{i}, x_{i+1}\right)$ at time $t^{n}$ and $t^{n+1}$, respectively.

Let at time $t^{n}$ the initial vector-valued function $\boldsymbol{f}_{i+1 / 2}=(u, p, \rho, Z)_{i+1 / 2}^{\top}$ be known at each 
zone mid point $x_{i+1 / 2}^{n}$, where, for instance, $u_{i+1 / 2}$ is the cell-average value of the velocity

$$
u_{i+1 / 2}=\frac{1}{h_{i+1 / 2}} \int_{x_{i}^{n}}^{x_{i+1}^{n}} u\left(x, t^{n}\right) \mathrm{d} x .
$$

Consider the control volume $\left(x_{i}, x_{i+1}\right)$ at time $t^{n}$ and $t^{n+1}$. We can draw conceptually the quadrilateral cell $\Omega_{i+1 / 2}^{n+1 / 2}$ in the $x$ - $t$ plane, see Fig. 2. Integrating the system (6) along the contour $\partial \Omega_{i+1 / 2}^{n+1 / 2}$, we obtain the system of the finite difference equations

$$
\begin{array}{r}
\boldsymbol{\sigma}^{i+1 / 2} h^{i+1 / 2}-\boldsymbol{\sigma}_{i+1 / 2} h_{i+1 / 2}-\boldsymbol{\sigma}_{i+1} h_{i+1}+\boldsymbol{\sigma}_{i} h_{i}+\Delta t\left(\boldsymbol{a}_{i+1}-\boldsymbol{a}_{i}\right)= \\
\frac{1}{2} \Delta t\left(h^{i+1 / 2}+h_{i+1 / 2}\right) \boldsymbol{c}_{i+1 / 2}^{n+1 / 2},
\end{array}
$$

where the value of $\boldsymbol{\sigma}^{i+1 / 2}$ is taken at point $x_{i+1 / 2}^{n+1}, \boldsymbol{\sigma}_{i}$ and $\boldsymbol{\sigma}_{i+1}$ at $x_{i}^{n+1 / 2}$ and $x_{i+1}^{n+1 / 2}$, respectively; $h_{i}=x_{i}^{n+1}-x_{i}^{n}$ is the projection of the lateral edge $x_{i}^{n} x_{i}^{n+1}$ (further referred to as the $i$ th lateral edge) onto the $x$-axis. The function $\boldsymbol{c}_{i+1 / 2}^{n+1 / 2}$ is taken in the center of the quadrilateral cell. The Eqs. (9) can be interpreted as a discrete form of the conservation laws on the moving mesh. Actually, the change of the vector-valued function $\boldsymbol{\sigma}$ in the control volume $\left(x_{i}, x_{i+1}\right)$ within time $\Delta t$ (first two terms in the left-hand side of (9)) is due the following: motion of the segment endpoints $x_{i}$ and $x_{i+1}$ (third and fourth terms); convection (fifth term); and internal source (right-hand side term).

To update the cell average values $\boldsymbol{f}^{i+1 / 2}$ via (9) with the second order of accuracy one needs to know the fluxes through the endpoints, in other words the values $\boldsymbol{f}_{i}, \boldsymbol{f}_{i+1}$ at time $t^{n+1 / 2}$. We will apply the predictor-corrector procedure. Besides, one assumes the vectorvalued discrete function $\boldsymbol{f}$ to be piece-wise linear within the control volume $\left(x_{i}, x_{i+1}\right)$.

At the first stage, predictor, in (9) we set $\boldsymbol{f}_{i}=\boldsymbol{f}_{1, i+1 / 2}$, and $\boldsymbol{f}_{i+1}=\boldsymbol{f}_{2, i+1 / 2}$, i.e. take those values at the lateral edges at time $t^{n}$ (superscript $n$ is omitted) instead of at $t^{n+1 / 2}$. To find the values at the left and right zone ends, i.e., $\boldsymbol{f}_{1, i+1 / 2}$, and $\boldsymbol{f}_{2, i+1 / 2}$ (see Fig. 2), we use the interpolation involving a monotonicity procedure $[2,5]$. Note that many interpolation procedures can be used here, for instance, minmod [38] reformulated on the nonuniform mesh. All of them provide rather similar results for the discontinuous solutions (especially on the curvilinear mesh in the two-dimensional case). Actually we find some "effective" derivatives $\delta \boldsymbol{f}_{i+1 / 2}$ which are used to calculate the values at the zone ends

$$
\boldsymbol{f}_{1, i+1 / 2}=\boldsymbol{f}_{i+1 / 2}-0.5 \delta \boldsymbol{f}_{i+1 / 2} h_{i+1 / 2}, \quad \boldsymbol{f}_{2, i+1 / 2}=\boldsymbol{f}_{i+1 / 2}+0.5 \delta \boldsymbol{f}_{i+1 / 2} h_{i+1 / 2} .
$$

We also set $\boldsymbol{c}_{i+1 / 2}^{n+1 / 2}=\boldsymbol{c}_{i+1 / 2}^{n}$. Therefore, the intermediate values $\overline{\boldsymbol{f}}^{i+1 / 2}$ at the $n+1$ th level can be updated using (9).

Next, at the second stage, corrector, we obtain the pre-wave values of $\boldsymbol{f}$ at the lateral 
edges $i$ and $i+1$ at time $t^{n+1 / 2}$. With this purpose we set the effective derivatives at $t^{n+1}$ equal to the ones at $t^{n}[45]$, i.e. $\delta \overline{\boldsymbol{f}}^{i+1 / 2}=\delta \boldsymbol{f}_{i+1 / 2}$. Then at $t^{n+1}$ the values at the zone ends for the intermediate function $\overline{\boldsymbol{f}}^{i+1 / 2}$ are obtained from the relations similar to (10). Taking the mean of the zone ends values at $t^{n}$ and $t^{n+1}$ we obtain the pre-wave states at the lateral edges $i$ and $i+1$ at time $t^{n+1 / 2}$ as follows:

$$
\begin{aligned}
\boldsymbol{f}_{1, i+1 / 2}^{n+1 / 2} & =0.5\left[\boldsymbol{f}_{i+1 / 2}+\overline{\boldsymbol{f}}^{i+1 / 2}-0.5 \delta \boldsymbol{f}_{i+1 / 2}\left(h_{i+1 / 2}+h^{i+1 / 2}\right)\right] \\
\boldsymbol{f}_{2, i+1 / 2}^{n+1 / 2} & =0.5\left[\boldsymbol{f}_{i+1 / 2}+\overline{\boldsymbol{f}}^{i+1 / 2}+0.5 \delta \boldsymbol{f}_{i+1 / 2}\left(h_{i+1 / 2}+h^{i+1 / 2}\right)\right] .
\end{aligned}
$$

Knowing the pre-wave values $\boldsymbol{f}_{2, i-1 / 2}^{n+1 / 2}$ and $\boldsymbol{f}_{1, i+1 / 2}^{n+1 / 2}$ from the both side of every point $x_{i}$, one obtains the endpoints values $\boldsymbol{f}_{i}^{n+1 / 2}$ and $\boldsymbol{f}_{i+1}^{n+1 / 2}$ by solving the Riemann problem (see section 2.4 below). Now we can calculate the values $\boldsymbol{\sigma}_{i}, \boldsymbol{\sigma}_{i+1}, \boldsymbol{a}_{i}$, and $\boldsymbol{a}_{i+1}$. Substituting them into Eqs. (9) gives the final values $\boldsymbol{f}^{i+1 / 2}$.

The above described scheme is second-order accurate in both space and time provided that the solution is smooth in the entire flow domain and the mesh is quasiuniform [2], i.e.

$$
h_{i+1 / 2}-h_{i-1 / 2}=\mathcal{O}\left(\left(h_{i+1 / 2}\right)^{2}\right)
$$

\subsection{Riemann problem on the moving mesh}

In the calculations we assume the detonation wave to be resolved by several grid points located in the burning zone. On the Rayleigh-Mikhel'son line the burning zone is enclosed between the points 1 and 2 (or 4 and 5), see Fig. 1. Thus, we work with the model corresponding to the real physical process, where the heating shock moves first and behind it is the burning zone. In this model, we can use the Riemann solver developed for the non-reactive gas, because at point 1 (or 4 ), immediately behind the precursor shock, the reaction has not taken place yet.

We apply the Riemann solver developed by Prokopov [31] based on the exact solution of the nonheating PDE system (for description of this Riemann solver see also [36]). The "exact solution" means that we determine accurately the wave pattern emerging after left-side and right-side gases (each with constant parameters) begin to interact.

To demonstrate how to take into account the grid nodes movement let us consider the $i$ th lateral edge of the cell $\Omega_{i+1 / 2}^{n+1 / 2}$ within the time interval from $t^{n+1 / 2}$ to $t^{n+1}$. Assume that after solving the Riemann problem at point $x_{i}^{n+1 / 2}$ we have the wave pattern depicted in Fig. 3. There are 5 cases for the location of the segment $\left(x_{i}^{n+1 / 2}, x_{i}^{n+1}\right)$, or the upper half of the $i$ th lateral edge, in the wave pattern depending on the velocity $w_{i}$ of the $i$ th node. 
As for the post-wave values $\boldsymbol{f}_{i}^{n+1 / 2}$ (except $Z_{i}^{n+1 / 2}$ ), we take:

1. $\boldsymbol{f}_{i}^{n+1 / 2}=\boldsymbol{f}_{2, i-1 / 2}^{n+1 / 2} \quad$ if $\quad w_{i}<d_{\mathrm{sh}}$, where $d_{\mathrm{sh}}$ is the speed of the left shock.

2. $\boldsymbol{f}_{i}^{n+1 / 2}=\boldsymbol{f}_{2}$ if $d_{\mathrm{sh}}<w_{i}<d_{\text {cont }}$, where the vector $\boldsymbol{f}_{2}$ defines the flow parameters behind the shock, $d_{\text {cont }}$ is the speed of the contact discontinuity which equals to the velocity $u$ in that domain.

3. $\boldsymbol{f}_{i}^{n+1 / 2}=\boldsymbol{f}_{3}$ if $\quad d_{\text {cont }}<w_{i}<d_{\text {rar }}^{\text {lft }}$, where the vector $\boldsymbol{f}_{3}$ defines the parameters in the domain between the contact discontinuity and left characteristic of the rarefaction wave expanding with the speed $d_{\mathrm{rar}}^{\mathrm{lft}}$.

4. $\boldsymbol{f}_{i}^{n+1 / 2}=\phi(x / t) \quad$ if $\quad d_{\mathrm{rar}}^{\mathrm{lft}}<w_{i}<d_{\mathrm{rar}}^{\mathrm{rght}}$, i.e. we calculate the flow parameters in the rarefaction wave using the similarity variable $x / t$. Here $d_{\text {rar }}^{\text {rght }}$ is the speed of the right characteristic in the rarefaction fan.

5. $\boldsymbol{f}_{i}^{n+1 / 2}=\boldsymbol{f}_{1, i+1 / 2}^{n+1 / 2} \quad$ if $\quad w_{i}>d_{\mathrm{rar}}^{\mathrm{rght}}$

Note that in [31] the above algorithm is applied at time $t^{n}$.

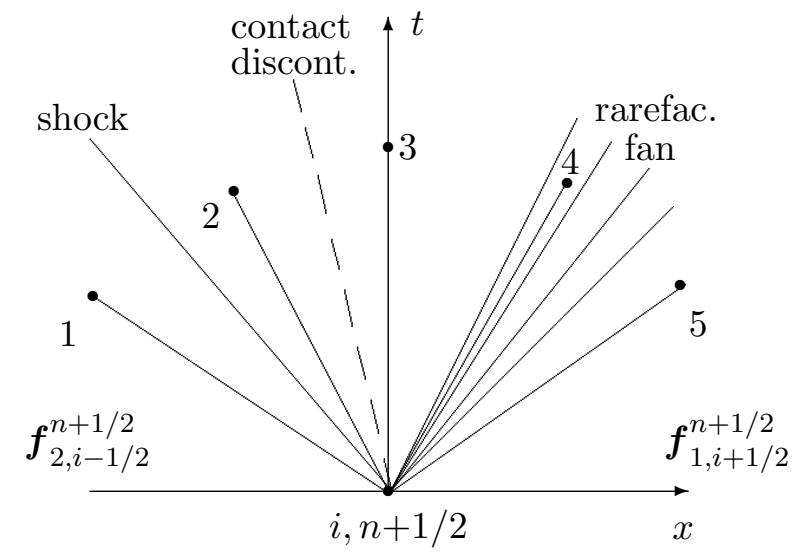

Figure 3. Five possible cases of location of the segment $\left(x_{i}^{n+1 / 2}, x_{i}^{n+1}\right)$ in the wave pattern. Points $1, \ldots, 5$ indicate location of the $i$ th node at $t^{n+1}$.

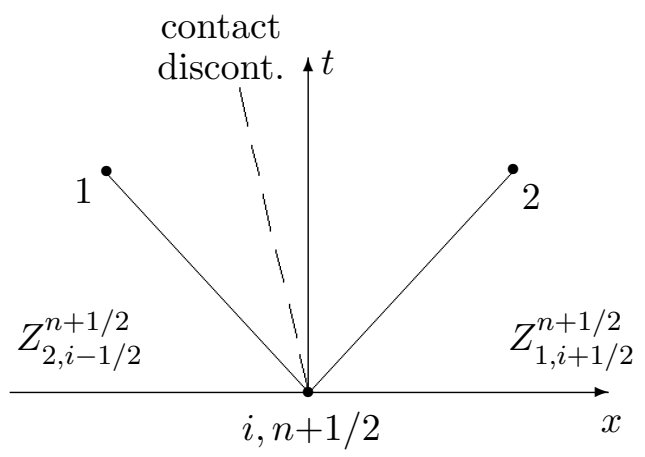

Figure 4. Two possible cases of location of the segment $\left(x_{i}^{n+1 / 2}, x_{i}^{n+1}\right)$ in the wave pattern for the chemical reaction equation.

For the reactive equation, since the characteristic is $\mathrm{d} x / \mathrm{d} t=u$, we use the jump condition 
to find the post-wave value $Z_{i}^{n+1 / 2}$, see Fig. 4, namely

$$
Z_{i}^{n+1 / 2}=\left\{\begin{array}{lc}
Z_{2, i-1 / 2}^{n+1 / 2} & \text { if } w_{i}<d_{\text {cont }} \\
Z_{1, i+1 / 2}^{n+1 / 2} & \text { otherwise }
\end{array}\right.
$$

In the center of the quadrilateral cell we approximate the flow parameters by

$$
\boldsymbol{f}_{i+1 / 2}^{n+1 / 2}=0.5\left(\boldsymbol{f}_{i+1 / 2}+\overline{\boldsymbol{f}}^{i+1 / 2}\right)
$$

At last, we update the final values $\boldsymbol{f}^{i+1 / 2}$ at time $t^{n+1}$ by using (9).

\subsection{Stability condition}

In the one-dimensional case the choice of the admissible time step $\Delta t$ has a clear physical sense. On the moving grid $\Delta t$ is given by

$$
\Delta t=c_{\mathrm{cfl}} \min _{i} \Delta t_{i+1 / 2}
$$

where in every cell the local time step is determined by (cf. [31])

$$
\Delta t_{i+1 / 2}=\frac{h_{i+1 / 2}}{\max \left(d_{i}^{\mathrm{rght}}-w_{i+1},-d_{i+1}^{\mathrm{ftt}}+w_{i}\right)}
$$

Here $d_{i}^{\text {rght }}$ and $d_{i+1}^{\text {lft }}$ are the extreme right and left wave speeds at points $x_{i}$ and $x_{i+1}$, respectively, obtained by solving the Riemann problem, and $w_{i}$ is the velocity of the node $x_{i}$. The condition (14) means that we estimate the time within which the left characteristic (in the linearized analysis this is a straight line), emanating from the $i+1$ th node, achieves the $i$ th node, as well as the time within which the right characteristic, emanating from the $i$ th node, achieves the $i+1$ th node. From these two time steps we take the minimal. If in some cell $\Delta t_{i+1 / 2}<0$ (on the moving mesh it can happen), we exclude this cell from consideration in (13). The condition (14) covers the stability condition for the kinetic equation as well, because here the characteristic extends with the speed of the contact discontinuity. The CFL number $c_{\mathrm{cfl}}$ (or coefficient of reserve [31]) is a correction to the non-linearity of the PDE system. To calculate the node velocity $w_{i}$, on one hand it is necessary to know the time step $\Delta t$, and on the other hand $w_{i}$ participates in determining $\Delta t$. By this reason at time level $n+1$ we use $\Delta t$ obtained at the preceding level $n$. The coefficient $c_{\mathrm{cfl}}<1$, usually about 0.5 , may be corrected during the computation. 
In the linearized analysis on the fixed grid we use a sound approach to get $\Delta t_{i+1 / 2}$ in each cell

$$
\Delta t_{i+1 / 2}=\frac{h_{i+1 / 2}}{\max \left(u_{i+1 / 2}+c_{i+1 / 2},-u_{i+1 / 2}+c_{i+1 / 2}\right)}
$$

where the sound speed is equal to $c_{i+1 / 2}^{2}=\gamma(p / \rho)_{i+1 / 2}$. The formula (15) can be also used if the mesh-moving speed is not too high.

Let us return to the matter on prescribing the boundary conditions at the end of the reaction zone. Besides the 'piston' condition, for instance in [37] for the linear stability analysis the 'radiation' condition is also applied. It requires that there is no perturbation to the steady state at the end of the reaction zone that travels forward, towards the detonation wave from the piston. This, in turn, implies that the third equation in (7) is excluded by assuming its linear dependence on the first and second ones. However, such a condition can not be used in nonlinear calculations for the following reason. Assuming the flow is smooth at the end of the reaction zone, the numerical scheme can be constructed by approximating the PDEs in the nondivergent form (7) and, therefore, by treating (8). Now $\rho_{o}$ and $c_{o}$ are the frozen coefficients in the numerical procedure defined by initial data at the predictor and corrector steps and depending on the coordinates $x, y$. We can not eliminate the invariant $p+\rho_{o} c_{o} u$ influence on the forward solution. It would mean the third equation be excluded from the system (8). Besides, the overcompressed steady detonation mode is only possible under the support provided by this invariant [58]. The unstable detonation mode, which will be considered in sections 5.2 and 5.3 , is only a deviation of the basic steady state. Thus, one can only prescribe the invariant value at the left endpoint. Due to the truncation errors this invariant always transfers pertrubation to the forward state. The above analysis is valid also when the third equation of (7) is inhomogeneous with the chemical term in the right-hand part.

\section{Two-dimensional case}

\subsection{System of equations}

The governing system of the differential equations relating to the two-dimensional reactive gas flow is

$$
\frac{\partial \boldsymbol{\sigma}}{\partial t}+\frac{\partial \boldsymbol{a}}{\partial x}+\frac{\partial \boldsymbol{b}}{\partial y}=\boldsymbol{c}
$$

where $\boldsymbol{\sigma}=(\rho, \rho u, \rho v, E, \rho Z)^{\top}, \boldsymbol{a}=\left(\rho u, \rho u^{2}+p, \rho u v, u(E+p), \rho u Z\right)^{\top}$,

$\boldsymbol{b}=\left(\rho v, \rho u v, \rho v^{2}+p, v(E+p), \rho v Z\right)^{\top}, \boldsymbol{c}=(0,0,0,0,-\rho K(T) Z)^{\top}, u$ and $v$ are the velocity 
components. Now the total energy becomes $E=\rho\left[e+0.5\left(u^{2}+v^{2}\right)\right]+q_{o} \rho Z$.

We perform calculations utilizing the integral conservation laws which can be derived by integrating the system (16) and transforming the volume integrals in $\mathbb{R}^{3}$ space $(x, y, t)$ to the surface integrals with the use of the Gauss's theorem

$$
\iiint_{\Omega}\left[\frac{\partial \boldsymbol{\sigma}}{\partial t}+\frac{\partial \boldsymbol{a}}{\partial x}+\frac{\partial \boldsymbol{b}}{\partial y}-\boldsymbol{c}\right] d \Omega=\oiint_{\partial \Omega} \boldsymbol{\sigma} \mathrm{d} x \mathrm{~d} y+\boldsymbol{a} \mathrm{d} y \mathrm{~d} t+\boldsymbol{b} \mathrm{d} t \mathrm{~d} x-\iiint_{\Omega} \boldsymbol{c} \mathrm{d} x \mathrm{~d} y \mathrm{~d} t=0 .
$$

Here the domain $\Omega$ with the boundary $\partial \Omega$ is a homeomorphic sphere in space $(x, y, t)$, see Fig. 5. Hence, the system can be rewritten in the integral form as follows (such generalized formulation for the nonreactive gas flow has been suggested in [31])

$$
\oiint_{\partial \Omega} \boldsymbol{\sigma} \mathrm{d} x \mathrm{~d} y+\boldsymbol{a} \mathrm{d} y \mathrm{~d} t+\boldsymbol{b} \mathrm{d} t \mathrm{~d} x=\iiint_{\Omega} \boldsymbol{c} \mathrm{d} x \mathrm{~d} y \mathrm{~d} t .
$$
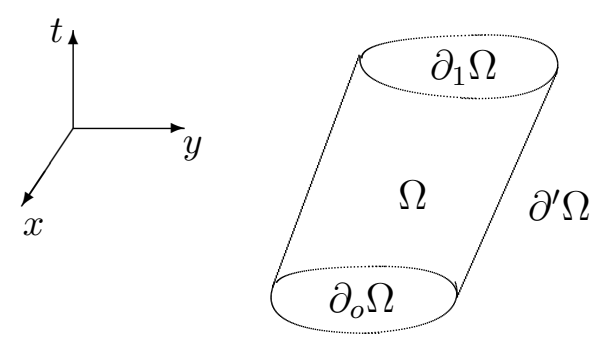

Figure 5. Domain $\Omega$ in space $(x, y, t)$ with the boundary $\partial \Omega=\partial_{o} \Omega \cup \partial_{1} \Omega \cup \partial^{\prime} \Omega$. Here $\partial_{o} \Omega$ and $\partial_{1} \Omega$ is the underlying control volume in the $x-y$ plane at time $t_{o}$ and $t_{1}$, respectively.

The meaning of this form is the following. The boundary $\partial \Omega$ of the underlying domain consists of three parts: $\partial \Omega=\partial_{o} \Omega \cup \partial_{1} \Omega \cup \partial^{\prime} \Omega$. The amount of the parameters (mass, momentum, total energy, and reactant mass) in the two-dimensional domain, a control volume $\partial_{o} \Omega$, at time $t_{o}$ is equal to

$$
\iint_{\partial_{o} \Omega} \boldsymbol{\sigma} \mathrm{d} x \mathrm{~d} y
$$

Since the control volume boundary moves, by time $t_{1}$ the domain $\partial_{o} \Omega$ becomes $\partial_{1} \Omega$ and the corresponding amount changes to

$$
\iint_{\partial_{1} \Omega} \boldsymbol{\sigma} \mathrm{d} x \mathrm{~d} y
$$

The change of the parameters is due to the flux through the moving boundary of the control volume and the surface stresses (to the momentum and energy equations), which can be expressed as follows:

$$
\oint_{C} \boldsymbol{a} \mathrm{d} y+\boldsymbol{b} \mathrm{d} x
$$


Here the contour $C$ is the boundary of the two-dimensional control volume in the $x-y$ plane, which changes with time. At $t=t_{o}$ the contour $C$ is the boundary of the control volume $\partial_{o} \Omega$ and at $t=t_{1}$ of $\partial_{1} \Omega$. The change of the parameters within the time $\left(t_{o}, t_{1}\right)$ is

$$
\int_{t_{0}}^{t_{1}} \oint_{C}(\boldsymbol{a} \mathrm{d} y+\boldsymbol{b} \mathrm{d} x) \mathrm{d} t=\iint_{\partial^{\prime} \Omega}(\boldsymbol{a} \mathrm{d} y+\boldsymbol{b} \mathrm{d} x) \mathrm{d} t .
$$

Furthermore, we should take into account the reactant mass change due to burning, caused by the source term in the right-hand side of (16). To this end, we formulate the integral conservation laws as follows. The change of the flow parameters (mass, momentum, total energy, or reactant mass) in the control volume is due to the flux through the moving boundary of the control volume, surface stresses, and internal sources

$$
\iint_{\partial_{1} \Omega} \boldsymbol{\sigma} \mathrm{d} x \mathrm{~d} y-\iint_{-\partial_{o} \Omega} \boldsymbol{\sigma} \mathrm{d} x \mathrm{~d} y+\iint_{\partial^{\prime} \Omega}(\boldsymbol{a} \mathrm{d} y+\boldsymbol{b} \mathrm{d} x) \mathrm{d} t=\iiint_{\Omega} \boldsymbol{c} \mathrm{d} x \mathrm{~d} y \mathrm{~d} t,
$$

where the sign "-" with $\partial_{o} \Omega$ indicates the change of the domain orientation. This system can be rewritten in the brief form (17). The integral conservation laws extends the class of admissible functions since it is not required that the solution to be differentiable as in (16), and it governs the discontinuous solutions as well.

Of course, logically we should begin the governing equations derivation with the integral form (17), since the system (16) is a consequence of (17). However, the form (16) is more habitual and that is why it is presented first.

\subsection{Numerical scheme}

We introduce the curvilinear moving grid in the $x$-y plane, and the $(i+1 / 2, j+1 / 2)$ th cell at time $t^{n}$ and $t^{n+1}$ is shown Fig. 6 . We draw mentally a domain $\Omega$ in $\mathbb{R}^{3}$ space $(x, y, t)$, being a hexahedron with planar top and bottom faces and four ruled lateral faces.

The bottom face of the hexahedron $\Omega$ is the control volume at time $t^{n}$ and the top face at $t^{n+1}$. Integrating (17) over the oriented surface, i.e. the boundary $\partial \Omega$ of the hexahedron, gives a cell-centered finite-volume discretization of the governing equations

$$
\begin{array}{r}
\boldsymbol{\sigma}^{i+1 / 2, j+1 / 2} A_{1^{\prime} 2^{\prime} 3^{\prime} 4^{\prime}}-\boldsymbol{\sigma}_{i+1 / 2, j+1 / 2} A_{1234}+\boldsymbol{Q}_{411^{\prime} 4^{\prime}}+\boldsymbol{Q}_{233^{\prime} 2^{\prime}}+\boldsymbol{Q}_{122^{\prime} 1^{\prime}}+\boldsymbol{Q}_{344^{\prime} 3^{\prime}}= \\
\boldsymbol{c}_{i+1 / 2, j+1 / 2}^{n+1 / 2} \Omega
\end{array}
$$

where $\boldsymbol{\sigma}^{i+1 / 2, j+1 / 2}$ and $\boldsymbol{\sigma}_{i+1 / 2, j+1 / 2}$ are the average values at time $t^{n+1}$ and $t^{n}$ in the center of the top and bottom faces, respectively; $A_{1^{\prime} 2^{\prime} 3^{\prime} 4^{\prime}}$ and $A_{1234}$ are the areas of the 


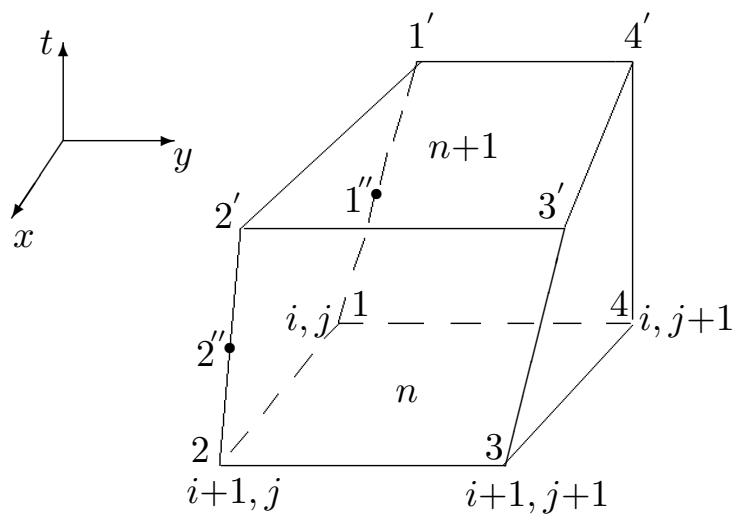

Figure 6. Hexahedron $\Omega$ in $\mathbb{R}^{3}$ space with bottom (1234) and top (1'2'3'4') faces, being the cell of the $2 \mathrm{D}$ moving mesh at time $t^{n}$ and $t^{n+1}$, respectively.

corresponding faces. Each of the four vector values $\boldsymbol{Q}_{411^{\prime} 4^{\prime}}, \boldsymbol{Q}_{233^{\prime} 2^{\prime}}, \boldsymbol{Q}_{122^{\prime} 1^{\prime}}$ and $\boldsymbol{Q}_{344^{\prime} 3^{\prime}}$ is the amount of the mass, momentum, energy, and reactant mass which flows into and out the quadrilateral cell 1234 within time $\Delta t$ through the corresponding moving edges of the cell. The value $\boldsymbol{c}_{i+1 / 2, j+1 / 2}^{n+1 / 2}$ is defined in the center of the hexahedron (in other words in the center of the quadrilateral cell at $t^{n+1 / 2}$ ), and $\Omega$ is the hexahedron volume.

For example, $\boldsymbol{Q}_{122^{\prime} 1^{\prime}}$, the change of the parameters due to the flux through the edge 12 within time $\Delta t$, is given by

$$
\boldsymbol{Q}_{122^{\prime} 1^{\prime}}=\boldsymbol{\sigma}_{i+1 / 2, j}^{n+1 / 2} A_{122^{\prime} 1^{\prime}}^{x y}+\boldsymbol{a}_{i+1 / 2, j}^{n+1 / 2} A_{122^{\prime} 1^{\prime}}^{y t}+\boldsymbol{b}_{i+1 / 2, j}^{n+1 / 2} A_{122^{\prime} 1^{\prime}}^{t x}
$$

where $\boldsymbol{\sigma}_{i+1 / 2, j}^{n+1 / 2}, \boldsymbol{a}_{i+1 / 2, j}^{n+1 / 2}$, and $\boldsymbol{b}_{i+1 / 2, j}^{n+1 / 2}$ are calculated using the parameters $\boldsymbol{f}=(u, v, p, \rho)^{\top}$ in the center of the face $122^{\prime} 1^{\prime}$, i.e. at the mid-point of edge 12 at time $t^{n+1 / 2}$ (or at the mid-point of edge $\left.1^{\prime \prime} 2^{\prime \prime}\right) ; A_{122^{\prime} 1^{\prime}}^{x y}, A_{122^{\prime} 1^{\prime}}^{y t}, A_{122^{\prime} 1^{\prime}}^{t x}$ are the areas of the projections of the face $122^{\prime} 1^{\prime}$ onto the coordinate planes $x-y, y$ - $t$, and $t$ - $x$, respectively, given by

$$
\begin{aligned}
& A_{122^{\prime} 1^{\prime}}^{x y}=\iint_{122^{\prime} 1^{\prime}} \mathrm{d} x \mathrm{~d} y=0.5\left[\left(x_{2^{\prime}}-x_{1}\right)\left(y_{1^{\prime}}-y_{2}\right)-\left(x_{1^{\prime}}-x_{2}\right)\left(y_{2^{\prime}}-y_{1}\right)\right] \\
& A_{122^{\prime} 1^{\prime}}^{y t}=\iint_{122^{\prime} 1^{\prime}} \mathrm{d} y \mathrm{~d} t=0.5 \Delta t\left(y_{2^{\prime}}+y_{2}-y_{1}-y_{1^{\prime}}\right) \\
& A_{122^{\prime} 1^{\prime}}^{t x}=\iint_{122^{\prime} 1^{\prime}} \mathrm{d} t \mathrm{~d} x=-0.5 \Delta t\left(x_{2^{\prime}}+x_{2}-x_{1}-x_{1^{\prime}}\right) .
\end{aligned}
$$

These expressions are obtained from the formula for the quadrangle 1234

$$
A_{1234}=A\left(x_{1}, y_{1} ; x_{2}, y_{2} ; x_{3}, y_{3} ; x_{4}, y_{4}\right)=0.5\left[\left(x_{3}-x_{1}\right)\left(y_{4}-y_{2}\right)-\left(x_{4}-x_{2}\right)\left(y_{3}-y_{1}\right)\right]
$$

when passing its contour in an anticlock-wise manner. 
As in the one-dimensional case, the values $\boldsymbol{f}^{i+1 / 2, j+1 / 2}$ are updated by two stages using a predictor-corrector procedure. At the first stage, predictor, we compute the intermediate values at the $n+1$ th level $\overline{\boldsymbol{f}}^{i+1 / 2, j+1 / 2}$ by using (18).

Let us consider the curvilinear coordinate $\xi$. Assume the function $\boldsymbol{f}$ to be linear within the cell $(i+1 / 2, j+1 / 2)$ in the $\xi$-direction. The values $\boldsymbol{f}_{i, j+1 / 2}$ and $\boldsymbol{f}_{i+1, j+1 / 2}$, specified at the left and right ends of the segment $((i, j+1 / 2),(i+1, j+1 / 2))$ at $t^{n}$, are defined via

$$
\begin{aligned}
\boldsymbol{f}_{i, j+1 / 2} & =\boldsymbol{f}_{i+1 / 2, j+1 / 2}-0.5 \delta \boldsymbol{f}_{i+1 / 2} h_{i+1 / 2} \\
\boldsymbol{f}_{i+1, j+1 / 2} & =\boldsymbol{f}_{i+1 / 2, j+1 / 2}+0.5 \delta \boldsymbol{f}_{i+1 / 2} h_{i+1 / 2} .
\end{aligned}
$$

Here $\delta \boldsymbol{f}_{i+1 / 2}$ is the "effective" derivative in the $\xi$-direction, and the spacing $h_{i+1 / 2}$ is the length of the underlying segment. Note that $\delta \boldsymbol{f}_{i+1 / 2}$ and $h_{i+1 / 2}$ are the short notations for $\left(\delta \boldsymbol{f}_{\xi}\right)_{i+1 / 2, j+1 / 2}$ and $\left(h_{\xi}\right)_{i+1 / 2, j+1 / 2}$, respectively. When determining $\delta \boldsymbol{f}_{i+1 / 2}$, to suppress spurious oscillations in the vicinity of discontinuities the monotonicity algorithm should be applied as that of in the one-dimensional case. The spacing $h_{i+1 / 2}$ is given by

$$
h_{i+1 / 2}=0.5 \sqrt{\left(x_{i+1, j}^{n}+x_{i+1, j+1}^{n}-x_{i, j}^{n}-x_{i, j+1}^{n}\right)^{2}+\left(y_{i+1, j}^{n}+y_{i+1, j+1}^{n}-y_{i, j}^{n}-y_{i, j+1}^{n}\right)^{2}} .
$$

By analogy we calculate the values $\boldsymbol{f}_{i+1 / 2, j}$ and $\boldsymbol{f}_{i+1 / 2, j+1}$, which are specified at the left and right ends of the segment in the $\eta$-direction in the cell. Note, since we interpolate $\boldsymbol{f}$ along the curvilinear coordinate lines $\xi$ and $\eta$, in general, the order of interpolation is less then 2 , and equal 2 only if the mesh is rectangular.

We substitute the determined values of $\boldsymbol{f}$ at the mid-point of the lateral edge 12 of the quadrilateral 1234, i.e. at time $t^{n}$ instead of the ones at time $t^{n+1 / 2}$, in (19) to find the values $\boldsymbol{Q}_{122^{\prime} 1^{\prime}}$. The values $\boldsymbol{Q}_{411^{\prime} 4^{\prime}}, \boldsymbol{Q}_{233^{\prime} 2^{\prime}}$, and $\boldsymbol{Q}_{344^{\prime} 3^{\prime}}$ can be found in a similar way. For the parameter $\boldsymbol{c}_{i+1 / 2, j+1 / 2}^{n+1 / 2}$ we use the one at time $t^{n}$. A good approximation to the volume $\Omega$ is given by

$$
\Omega=0.5\left(A_{1^{\prime} 2^{\prime} 3^{\prime} 4^{\prime}}+A_{1234}\right) \Delta t .
$$

Finally, from (18) we obtain the intermediate values $\overline{\boldsymbol{f}}^{i+1 / 2, j+1 / 2}$ at the $n+1$ th level.

We now discuss the second stage, corrector. As in the one-dimensional case we obtain the values in the center of the faces $122^{\prime} 1^{\prime}$ and $344^{\prime} 3^{\prime}$, i.e. at the mid-point of the edges 12 and 34 at time $t^{n+1 / 2}$ :

$$
\begin{aligned}
\boldsymbol{f}_{i, j+1 / 2}^{n+1 / 2} & =0.5\left[\boldsymbol{f}_{i+1 / 2, j+1 / 2}+\overline{\boldsymbol{f}}^{i+1 / 2, j+1 / 2}-0.5 \delta \boldsymbol{f}_{i+1 / 2}\left(h_{i+1 / 2}+h^{i+1 / 2}\right)\right], \\
\boldsymbol{f}_{i+1, j+1 / 2}^{n+1 / 2} & =0.5\left[\boldsymbol{f}_{i+1 / 2, j+1 / 2}+\overline{\boldsymbol{f}}^{i+1 / 2, j+1 / 2}+0.5 \delta \boldsymbol{f}_{i+1 / 2}\left(h_{i+1 / 2}+h^{i+1 / 2}\right)\right],
\end{aligned}
$$

where the spacing $h^{i+1 / 2}$ is the length of the segment $((i, j+1 / 2),(i+1, j+1 / 2))$ at time $t^{n+1}$. We can obtain $\boldsymbol{f}_{i+1 / 2, j}^{n+1 / 2}$ and $\boldsymbol{f}_{i+1 / 2, j+1}^{n+1 / 2}$ in a similar way. These four vector values 
are used as the pre-wave states in the center of the corresponding lateral faces of the hexahedron for the Riemann problem.

Let us consider the face $122^{\prime} 1^{\prime}$. To get the postwave states $\boldsymbol{f}^{n+1 / 2}$ in the center of this face (for brevity we omit subscripts), i.e. at the mid-point of the segment $\left(1^{\prime \prime}, 2^{\prime \prime}\right)$, we solve the Riemann problem with the pre-wave states $(r, p, \rho)^{n+1 / 2}$ at this point on both sides of the face (one state relates to the underlying hexahedron and the other to the hexahedron adjacent to the face $\left.122^{\prime} 1^{\prime}\right)$. Here $r^{n+1 / 2}$ is the normal component of the velocity to the segment $\left(1^{\prime \prime}, 2^{\prime \prime}\right)$. We also use the tangential components of the velocity $q^{n+1 / 2}$ on those sides. The normal and tangential components of the velocity are given by

$$
r^{n+1 / 2}=n_{x} u^{n+1 / 2}+n_{y} v^{n+1 / 2}, \quad q^{n+1 / 2}=n_{y} u^{n+1 / 2}-n_{x} v^{n+1 / 2},
$$

where $n_{x}, n_{y}$ are the components of the outward unit normal vector to the segment $\left(1^{\prime \prime}, 2^{\prime \prime}\right)$.

After solving the Riemann problem, the post-wave values $(r, p, \rho)_{R}^{n+1 / 2}$ in the face center are defined. The post-wave tangential component of the velocity $q_{R}^{n+1 / 2}$ is given by

$$
q_{R}^{n+1 / 2}= \begin{cases}q^{n+1 / 2} & \text { if } w_{12} \leq d_{\text {cont }} \\ \tilde{q}^{n+1 / 2} & \text { otherwise }\end{cases}
$$

where $d_{\text {cont }}$ is the contact discontinuity speed in the Riemann problem, $w_{12}$ is the velocity of the edge 12 in the normal direction to this edge, and $\tilde{q}^{n+1 / 2}$ is the pre-wave tangential component of the velocity in the hexahedron adjacent to the face $122^{\prime} 1^{\prime}$. This condition expresses the fact that the tangential component of the velocity is discontinuous across the tangential discontinuity, cf. [18]. The velocity $w_{12}$ can be derived from the equality

$$
\Delta t l_{1^{\prime \prime} 2^{\prime \prime}} w_{12}=A_{122^{\prime} 1^{\prime}}^{x y},
$$

where $l_{1^{\prime \prime}} 2^{\prime \prime}$ is the length of the segment $\left(1^{\prime \prime}, 2^{\prime \prime}\right)$. Next we restore the Cartesian components of the post-wave velocity in the center of the face $122^{\prime} 1^{\prime}$

$$
u_{R}^{n+1 / 2}=n_{x} r_{R}^{n+1 / 2}+n_{y} q_{R}^{n+1 / 2}, \quad v_{R}^{n+1 / 2}=n_{y} r_{R}^{n+1 / 2}-n_{x} q_{R}^{n+1 / 2} .
$$

We treat the mass fraction $Z$ using (12) and find $Z_{R}^{n+1 / 2}$ on this face.

Given the post-wave values $(u, v, p, \rho, Z)_{R}^{n+1 / 2}$ in the center of the face $122^{\prime} 1^{\prime}$, we calculate $\boldsymbol{Q}_{122^{\prime} 1^{\prime}}$ via (19). Similarly we treat the Riemann problem in the center of the other three faces to obtain $\boldsymbol{Q}_{411^{\prime} 4^{\prime}}, \boldsymbol{Q}_{233^{\prime} 2^{\prime}}$, and $\boldsymbol{Q}_{344^{\prime} 3^{\prime}}$. When calculating $\boldsymbol{c}_{i+1 / 2, j+1 / 2}^{n+1 / 2}$, the values of $\boldsymbol{f}_{i+1 / 2, j+1 / 2}^{n+1 / 2}$ are given by

$$
\boldsymbol{f}_{i+1 / 2, j+1 / 2}^{n+1 / 2}=0.5\left(\boldsymbol{f}_{i+1 / 2, j+1 / 2}+\overline{\boldsymbol{f}}^{i+1 / 2, j+1 / 2}\right) .
$$


The final values of $\boldsymbol{f}^{i+1 / 2, j+1 / 2}$ at time $t^{n+1}$ are obtained by using (18).

\subsection{Stability condition}

In the $2 \mathrm{D}$ case the choice of the admissible step $\Delta t$ may be estimated in the energetic norm to the underlying Eqs. (16), written as a t-hyperbolic by Friedrichs's system [31]. The step $\Delta t$ for nonreactive gas flow calculation in the $(i+1 / 2, j+1 / 2)$ th cell is given by

$$
\Delta t_{i+1 / 2, j+1 / 2}=\frac{\Delta t^{\prime} \Delta t^{\prime \prime}}{\Delta t^{\prime}+\Delta t^{\prime \prime}}
$$

where

$$
\begin{aligned}
& \Delta t^{\prime}=\frac{h^{\prime}}{\max \left(d_{14}^{\text {right }}-w_{23} ;-d_{23}^{\text {fft }}+w_{14}\right)}, \quad \Delta t^{\prime \prime}=\frac{h^{\prime \prime}}{\max \left(d_{12}^{\text {right }}-w_{34} ;-d_{34}^{\text {fft }}+w_{12}\right)}, \\
& h^{\prime}=\frac{A_{1234}}{0.5 \sqrt{\left(x_{4}+x_{3}-x_{1}-x_{2}\right)^{2}+\left(y_{4}+y_{3}-y_{1}-y_{2}\right)^{2}}}, \\
& h^{\prime \prime}=\frac{A_{1234}}{0.5 \sqrt{\left(x_{3}+x_{2}-x_{4}-x_{1}\right)^{2}+\left(y_{3}+y_{2}-y_{4}-y_{1}\right)^{2}}} \text {. }
\end{aligned}
$$

Here $\Delta t^{\prime}$ and $\Delta t^{\prime \prime}$ are the admissible time steps to the one-dimensional scheme in the $\xi$ and $\eta$-direction, respectively; $h^{\prime}, h^{\prime \prime}$ are the "average heights" of the bottom face 1234 , and $w$ is the velocity of the corresponding cell edge. For example, $w_{12}$ is the velocity of the edge 12 in the normal direction determined via (22). Next, $d_{12}^{\text {right }}$ and $d_{14}^{\text {right }}$ are the "extreme right wave" speeds defined from solving the Riemann problem to the faces $122^{\prime} 1^{\prime}$

and $11^{\prime} 4^{\prime} 4$, respectively; $d_{23}^{\text {lft }}$ and $d_{34}^{\text {lft }}$ are the "extreme left wave" speeds to the faces $233^{\prime} 2^{\prime}$ and $433^{\prime} 4^{\prime}$, respectively.

The resulting time step over the mesh is given by

$$
\Delta t=c_{\mathrm{cfl}} \min _{i, j} \Delta t_{i+1 / 2, j+1 / 2}
$$

\subsection{Front tracking}

Now we turn to describe the front tracking procedure drawn in [31]. The front of the detonation wave is the precursor heating shock. Therefore, its velocity should be determined by using the Rankine-Hugoniot jump conditions, in other words from the Riemann problem solved on the cell edges located on the moving part of the domain boundary $\partial \Omega$ at time $t^{n+1 / 2}$. At the predictor stage we use the mesh taken from the preceding time level $t^{n}$ 


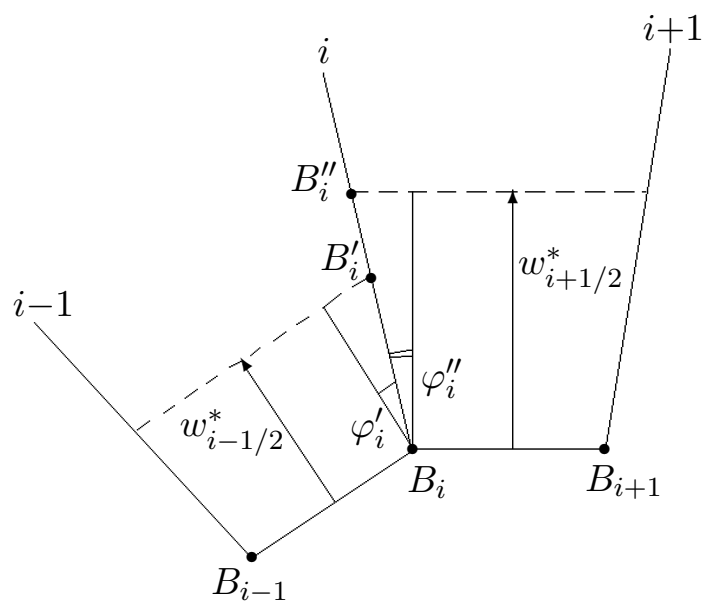

Figure 7. Front tracking for the heating shock wave.

Consider two those edges $B_{i-1} B_{i}$ and $B_{i} B_{i+1}$, see Fig. 7 . We must define the directions along which the boundary nodes $i-1, i, i+1$ move (for brevity we use only one subscript $i$ ). After solving the Riemann problem at the mid-point of these two edges we obtain the speed of extreme left (or right) characteristics, which are used as the normal velocities $w_{i-1 / 2}^{*}, w_{i+1 / 2}^{*}$ for these edges. The velocity of the $i$ th node along the $i$ th direction can be determined by two ways. First, as the velocity of the end-point $B_{i}$ in the edge $B_{i-1} B_{i}$

$$
w_{i}^{\prime}=\frac{w_{i-1 / 2}^{*}}{\cos \varphi_{i}^{\prime}}
$$

where $\varphi_{i}^{\prime}$ is the angle between the velocity vector $\boldsymbol{w}_{i-1 / 2}^{*}$ (that is a normal vector to the edge $\left.B_{i-1} B_{i}\right)$ and direction $i$. In Fig. 7 , the value of $w_{i}^{\prime}$ is indicated by the segment $\left(B_{i}, B_{i}^{\prime}\right)$. The second velocity of the $i$ th node along the $i$ th direction is determined as the velocity of the end-point $B_{i}$ in the edge $B_{i} B_{i+1}$

$$
w_{i}^{\prime \prime}=\frac{w_{i+1 / 2}^{*}}{\cos \varphi_{i}^{\prime \prime}}
$$

In Fig. 7, the value of $w_{i}^{\prime \prime}$ is indicated by the segment $\left(B_{i}, B_{i}^{\prime \prime}\right)$. As the velocity of the $i$ th node in the direction $i$ we use the interpolated value

$$
w_{i}^{*}=w_{i}^{\prime} \frac{l_{i+1 / 2}}{l_{i-1 / 2}+l_{i+1 / 2}}+w_{i}^{\prime \prime} \frac{l_{i-1 / 2}}{l_{i-1 / 2}+l_{i+1 / 2}},
$$

where

$$
l_{i+1 / 2}=\sqrt{\left(x_{i+1}^{n}-x_{i}^{n}\right)^{2}+\left(y_{i+1}^{n}-y_{i}^{n}\right)^{2}} .
$$

Afterwards the position of the $i$ th boundary node at time $t^{n+1}$ is given by

$$
x_{i}^{n+1}=x_{i}^{n}+w_{x, i}^{*} \Delta t, \quad y_{i}^{n+1}=y_{i}^{n}+w_{y, i}^{*} \Delta t
$$

where $w_{x, i}^{*}, w_{y, i}^{*}$ are the components of the velocity vector $\boldsymbol{w}_{i}^{*}$. 


\subsection{Discussion on accuracy}

As in the one-dimensional case this scheme is second-order accurate in both space and time provided that the solution is smooth in the entire flow domain and the mesh is quasiuniform and close to rectangular. In real applications, however, the smoothness of the flow is violated even if initially it was so. The flow parameters and their derivatives suffer discontinuity across the singularities: shock, contact discontinuity, and rarefaction wave extreme left and right characteristics. Thus, the linear interpolation (10) or (20), constructed via the Taylor expansion to the underlying functions, is generally incorrect for determining the fluxes at the cell boundaries, that is, the order of weak convergence falls in the entire flow domain. This effect was observed by Godunov at the end of 1950s [33]. The first order Godunov's scheme in the smooth domain of the rarefaction wave exhibited the weak convergence rate $r \approx 2 / 3$ instead of 1 as it is followed from the formal approximation of the system of equations. For high-order schemes, the reduction of accuracy in the smooth subdomains in the presence of shocks was also observed, for instance, in [40,12,22]. In [2], the present scheme was tested in the shock tube problem with the flow pattern consisting of the shock, contact discontinuity, and rarefaction fan. When tracking the extreme left and right characteristics and calculating within the rarefaction fan only, the convergence rate $r$ is 2 in the $L_{1}$ norm and is close to 2 in the $L_{\infty}$ norm. However, when calculating the entire flow domain with this scheme in the shock capturing manner, it falls down to 1 (in $L_{1}$ norm) even if to estimate $r$ only within the rarefaction wave, where the flow is smooth. From the standpoint of numerical calculations this effect can be explained as follows. In the vicinity of the singularity, due to the use of the limiter, in several cells (usually in two) the order of approximation falls down to 1 . This is because the piecewise linear reconstruction inside the cell is changed to piecewise constant. In addition, in one of these two cells the scheme may be even unconditionally unstable [45]. This leads to the disturbances from those cells that extend along the characteristics into subdomains of the smooth flow and they deteriorate the accuracy of the solution. Thus the term "second-order" is rather conditional. The remedy to support the high accuracy is to fit every shock by a special shock fitting procedure, which treat the discontinuities using the Rankine-Hugoniot jump conditions. Examples of such calculations will be presented in sections 5.2 and 5.3. One more way to prevent accuracy deterioration in the presence of shocks is to use the adaptive meshes. This effect will be discussed in section 5.3.

If the "second-order" scheme only yields first-order results, why use it? We keep in mind two reasons. The first one is obvious. The second-order error is significantly smaller than the first-order one. In [4], we performed calculations of the steady supersonic gas flow in the channel, where several oblique shocks divide the flow into subdomains with a known exact solution, by using four successively refined quasiuniform meshes. The present scheme exhibited the convergence rate $r=0.92$ to 0.96 , while the first-order scheme gave $r=0.35$ to 0.68 . The second reason is that we aim to use the adaptive mesh coupled with the flow solver. Treating the one-dimensional flow on the moving meshes with the first- and second- 
order schemes increases significantly the accuracy relatively to fixed mesh calculation [4]. In the two-dimensional flow, our scheme on the adaptive meshes increases the accuracy by factors up to 5 , while the first-order scheme does not provide enhancement at all.

\section{Grid generation}

The variational approach is employed to generate the moving adaptive mesh. It is from the class of so-called $r$-refinement methods. There is one more class of the adaptive mesh method, so called $h$-refinement, which is outside of the present study.

\subsection{Problem formulation}

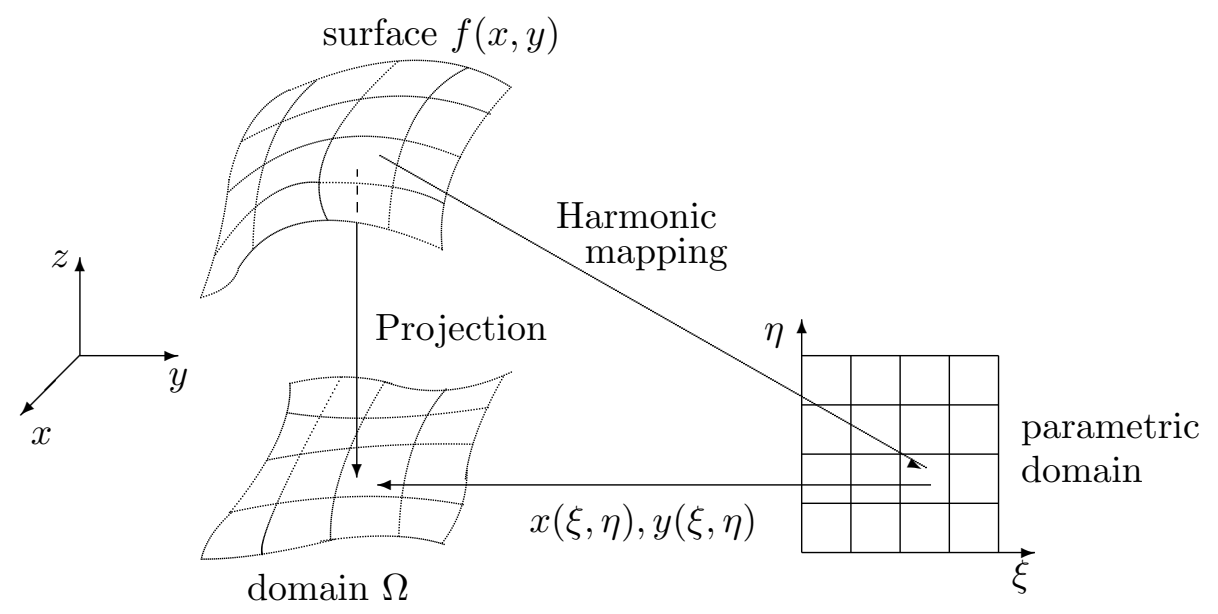

Figure 8. Adaptive grid generation.

The regular adaptive-harmonic mesh is constructed by minimizing the harmonic functional written for a surface of the control/monitor function $f(x, y)$ [39]. The relevant notations are shown in Fig. 8. The functional defining the adaptive grid, clustered in regions of large gradients of the function $f$, is (for derivation see $[14,4]$ )

$$
I=\iint \frac{\left(x_{\xi}^{2}+x_{\eta}^{2}\right)\left(1+f_{x}^{2}\right)+\left(y_{\xi}^{2}+y_{\eta}^{2}\right)\left(1+f_{y}^{2}\right)+2 f_{x} f_{y}\left(x_{\xi} y_{\xi}+x_{\eta} y_{\eta}\right)}{\left(x_{\xi} y_{\eta}-x_{\eta} y_{\xi}\right) \sqrt{1+f_{x}^{2}+f_{y}^{2}}} \mathrm{~d} \xi \mathrm{d} \eta .
$$

The problem of grid adaptation is formulated as follows. Let in the parametric domain the coordinates of the grid nodes be given, which is formed by square elements. Given the mapping of the parametric domain boundary onto the domain boundary $\partial \Omega$, we seek a harmonic mapping of the surface $S^{r 2}$ of the graph of $f(x, y)$ onto the parametric domain, by minimizing the functional (25). In the results we obtain the quasiuniform mesh on the surface $S^{r 2}$, which, being projected onto $\Omega$, defines the adaptive mesh in the physical domain $\Omega$. Subject to some known conditions, e.g. see [47], such a harmonic mapping exists 
and is a homeomorphism (one-to-one and onto). In unsteady problems such a formulation is considered at every time step.

In the 1-D case, to generate the inverse mapping of the graph of $f$ onto the segment on the parametric axis $\xi$ requires us to minimize the following functional (see [39]):

$$
I=\int \frac{1}{x_{\xi} \sqrt{1+f_{x}^{2}}} \mathrm{~d} \xi .
$$

With the purpose of controlling the degree of grid condensing in the domains of large gradients, it is convenient to use $c_{a} f$ instead of $f$, where $c_{a}$ is a coefficient of adaptation, which can depend on the variables $x, y$. Thus, we work with the control function multiplied by some coefficient $c_{a}$ in order to increase or decrease adaptation.

\subsection{Minimization of the functional}

The functional (25) is approximated in such a way that its minimum is attained on a grid of convex quadrilaterals, referred to as a convex grid [14,5],

$$
I^{h}=\sum_{i=1}^{i_{\max }} \sum_{k=1}^{4} \frac{1}{4}\left[F_{k}\right]_{i}
$$

where $F_{k}$ is the integrand evaluated in the $k$ th corner of the $i$ th cell. If the set of convex meshes is not empty, the system of the algebraic equations written at every interior node

$$
R_{x}=\frac{\partial I^{h}}{\partial x_{i}}=0, \quad R_{y}=\frac{\partial I^{h}}{\partial y_{i}}=0
$$

where $i$ is a global node number, has at least one solution that is a convex mesh. To find it an initial convex mesh should be given and an unconstrained minimization method needs to be used. Assuming the grid is convex at the $p$ th iteration step we find the coordinates of the $i$ th node at the $p+1$ th step using the quasi-Newton method (see $[14,5]$ for details):

$$
\begin{gathered}
x_{i}^{p+1}=x_{i}^{p}-\tau\left(R_{x} \frac{\partial R_{y}}{\partial y_{i}}-R_{y} \frac{\partial R_{x}}{\partial y_{i}}\right)\left(\frac{\partial R_{x}}{\partial x_{i}} \frac{\partial R_{y}}{\partial y_{i}}-\frac{\partial R_{y}}{\partial x_{i}} \frac{\partial R_{x}}{\partial y_{i}}\right)^{-1}, \\
y_{i}^{p+1}=y_{i}^{p}-\tau\left(R_{y} \frac{\partial R_{x}}{\partial x_{i}}-R_{x} \frac{\partial R_{y}}{\partial x_{i}}\right)\left(\frac{\partial R_{x}}{\partial x_{i}} \frac{\partial R_{y}}{\partial y_{i}}-\frac{\partial R_{y}}{\partial x_{i}} \frac{\partial R_{x}}{\partial y_{i}}\right)^{-1},
\end{gathered}
$$

where the iterative parameter is $0<\tau \leq 1$.

When generating a curvilinear mesh without adaptation in the physical domain $\Omega$ (if no adaptation, we substitute $f_{x}=f_{y}=0$ in (25)), the discrete functional (27) has an infinite 
barrier on the boundary of the set of convex grids $[13,14]$. This is caused by the condition of positiveness to the Jacobian of the mapping $J=x_{\xi} y_{\eta}-x_{\eta} y_{\xi}$. This property allows for generating the unfolded mesh in domains of any geometry. When adapting, due to discontinuities in the solution the infinite barrier disappears [4], which causes some grid cells to fold and modeling to break. To prevent it, first, we use a regularization procedure to the discrete functional [4]. The second way was suggested in [3] for unsteady problems. We specify $D_{\max }$, the maximal value of the modulus of the gradient of $f$, as follows

$$
D_{\max }=\lambda \max (|\nabla \tilde{f}|)
$$

where $\tilde{f}=c_{a} f^{h}, f^{h}$ is an interpolant of $f$, the coefficient $\lambda<1$ and $|\nabla \tilde{f}|=\sqrt{\tilde{f}_{x}^{2}+\tilde{f}_{y}^{2}}$. Next, the gradient of the function is updated via

$$
\nabla \tilde{f}^{*}=\left\{\begin{array}{cc}
D_{\max } \nabla \tilde{f} /|\nabla \tilde{f}| & \text { if }|\nabla \tilde{f}|>D_{\max } \\
\nabla \tilde{f} & \text { otherwise }
\end{array}\right.
$$

and the resulting values of $\tilde{f}_{x}^{*}$ and $\tilde{f}_{y}^{*}$ are substituted into (25) to replace $f_{x}$ and $f_{y}$.

In the one-dimensional case we minimize the following discrete functional for (26) (see [4] for details):

$$
I^{h}=\sum_{i=1}^{i_{\max }} \frac{\Delta \xi}{\left(x_{\xi}\right)_{i+1 / 2} \sqrt{1+\left(\tilde{f}_{x}\right)_{i+1 / 2}^{2}}}
$$

where the subscript $i+1 / 2$ means that the derivatives are determined in the mid point of the $i$ th spacing, by applying the Newton's method

$$
x_{i}^{p+1}=x_{i}^{p}-\tau \frac{\partial I^{h}}{\partial x_{i}}\left[\frac{\partial^{2} I^{h}}{\partial x_{i}^{2}}\right]^{-1} .
$$

\subsection{Boundary nodes redistribution}

In [4] it is demonstrated that the one- and two-dimensional discrete functionals are inconsistent, in the sense that the grid-nodes may move in a different way in the interior of the domain $\Omega$ and on its boundary $\partial \Omega$. Thus, when adapting, the cells near the boundary $\partial \Omega$ may degenerate. This happens when a shock hits the boundary $\partial \Omega$. To perform consistent redistribution of the grid nodes inside $\Omega$ and on $\partial \Omega$ it is suggested in [4] that the constrained minimization should be used. In this approach we minimize the following functional

$$
\tilde{I}^{h}=\sum_{i=1}^{i_{\max }} \sum_{k=1}^{4} \frac{1}{4}\left[F_{k}\right]_{i}+\sum_{l \in \mathcal{L}} \lambda_{l} G_{l}=I^{h}+\sum_{l \in \mathcal{L}} \lambda_{l} G_{l},
$$


where $I^{h}$ is the functional (27), the constraints $G_{l}=G\left(x_{l}, y_{l}\right)=0$ define the boundary $\partial \Omega$, $\lambda_{l}$ are the Lagrange multipliers, $\mathcal{L}$ is the set of the boundary nodes. If the set of convex grids is not empty, the system of the following algebraic equations has at least one solution being the convex mesh

$$
R_{x}=\frac{\partial I^{h}}{\partial x_{i}}+\lambda_{i} \frac{\partial G_{i}}{\partial x_{i}}=0, \quad R_{y}=\frac{\partial I^{h}}{\partial y_{i}}+\lambda_{i} \frac{\partial G_{i}}{\partial y_{i}}=0, \quad G_{i}=0
$$

Here $\lambda_{i}=0$ if $i \notin \mathcal{L}$ and constraints are defined at the boundary nodes $i \in \mathcal{L}$. The method of solving the system (33) is described in [4].

Note that using the constrained minimization without adaptation (when $f=$ const.) means that we seek the conformal mapping $x(\xi, \eta), y(\xi, \eta)$ of the parametric square onto the domain $\Omega$ with an additional parameter, so-called conformal modulus. This is because according to the Riemann theorem under the conformal mapping we can define correspondence only between three points on the boundary contour of the physical and parametric domains, and in our case there is a correspondence between four corner points on the boundary. That is why such a mesh is said to be rather a quasi-conformal grid, and, therefore, the mapping is quasi-conformal.

\subsection{Coupled algorithm}

Solving the one- or two-dimensional gas dynamics equations with grid adaptation at each time step contains the following stages:

(i) Generate the mesh at the next time level $t^{n+1}$.

(ii) Compute the gas dynamics values at time $t^{n+1}$.

(iii) Make one iteration to compute the new grid coordinates $(x, y)_{i}$ at $t^{n+1}$.

(iv) Repeat steps (ii) and (iii) using a given number of iterations.

(v) Compute the final gas dynamics values at $t^{n+1}$.

Note that in step (iv) in principle we should repeat the steps (ii) and (iii) up to convergence of the minimization procedure (29). But in this kind of problems we can not achieve convergence to the mesh within reasonable number of iterations. Moreover for the two-dimensional problems with discontinuous solution the discrete functional (27) has no minimum at all [4]. Nevertheless, the iterative procedure (29) allows us to condense significantly the grid lines towards the discontinuity and guarantee the grid to be unfolded.

Remark. After step (ii) we need to interpolate the gas dynamics parameters from the cell center to nodes. In the 1-D case the linear interpolation is employed. In the 2-D case an interpolation formula, which uses the cell area, is applied [5]. 


\section{$5 \quad$ Numerical results}

\subsection{One-dimensional Chapman-Jouguet detonation}

We consider the CJ detonation with the following gas parameters [17,7] (in CGS units)

$$
(u, p, \rho, Z)= \begin{cases}(u, p, \rho, Z)_{\mathrm{bnt}}=\left(4.162 \cdot 10^{4}, 6.270 \cdot 10^{6}, 1.945 \cdot 10^{-3}, 0\right) & \text { if } x \rightarrow-\infty \\ (u, p, \rho, Z)_{\mathrm{unb}}=\left(0,8.321 \cdot 10^{5}, 1.201 \cdot 10^{-3}, 1\right) & \text { if } x \rightarrow \infty\end{cases}
$$

where "bnt" represents the completely burnt gas and "unb" the unburnt gas with $\gamma=1.4$ and $R=1$. The heat release is $q_{o}=5.196 \cdot 10^{9}$. We use the kinetics model (5) when $\tau_{o}=1.717 \cdot 10^{-10}$, and $T_{\mathrm{ign}}=1.155 \cdot 10^{9}$. With these parameters the width of the reaction zone is approximately $5 \cdot 10^{-5}$. The mesh has $I=i_{\max }=100$ spacings and we set the rear boundary in 40 cells to the left from the point $x=0$ (corresponding to the peak coordinate of the ZND profile), and define there the transmissive boundary conditions to all flow parameters. On the front boundary we define $(u, p, \rho, Z)_{\mathrm{unb}}$.

In the first calculation we use the uniform mesh with $h=5 \cdot 10^{-6}$, which uses about 10 cells in the reaction zone with a time step $\Delta t=5 \cdot 10^{-12}$. The pressure and density profiles at time $t=10^{-8}$ are shown in Fig. 9 , and the corresponding error is presented in Table 1. The error is estimated within the interval $0.008<x<0.011$. Fig. 10 gives the results at $t=10^{-7}$ by using the coarse mesh with $h=5 \cdot 10^{-5}$ (only $\approx 1$ cell in the reaction zone) and $\Delta t=5 \cdot 10^{-11}$, which seem rather similar to the ones obtained in [7]. There is no spike in the ZND profile of the solution (in [7] a very small spike is observed, but we consider it as an insignificant difference) and the wave velocity is determined incorrectly. The reason is that on the under-resolved mesh hitting into the burning zone gives the front shift by one cell. This phenomenon has been discussed in a number of works, e.g. see $[28,17]$.
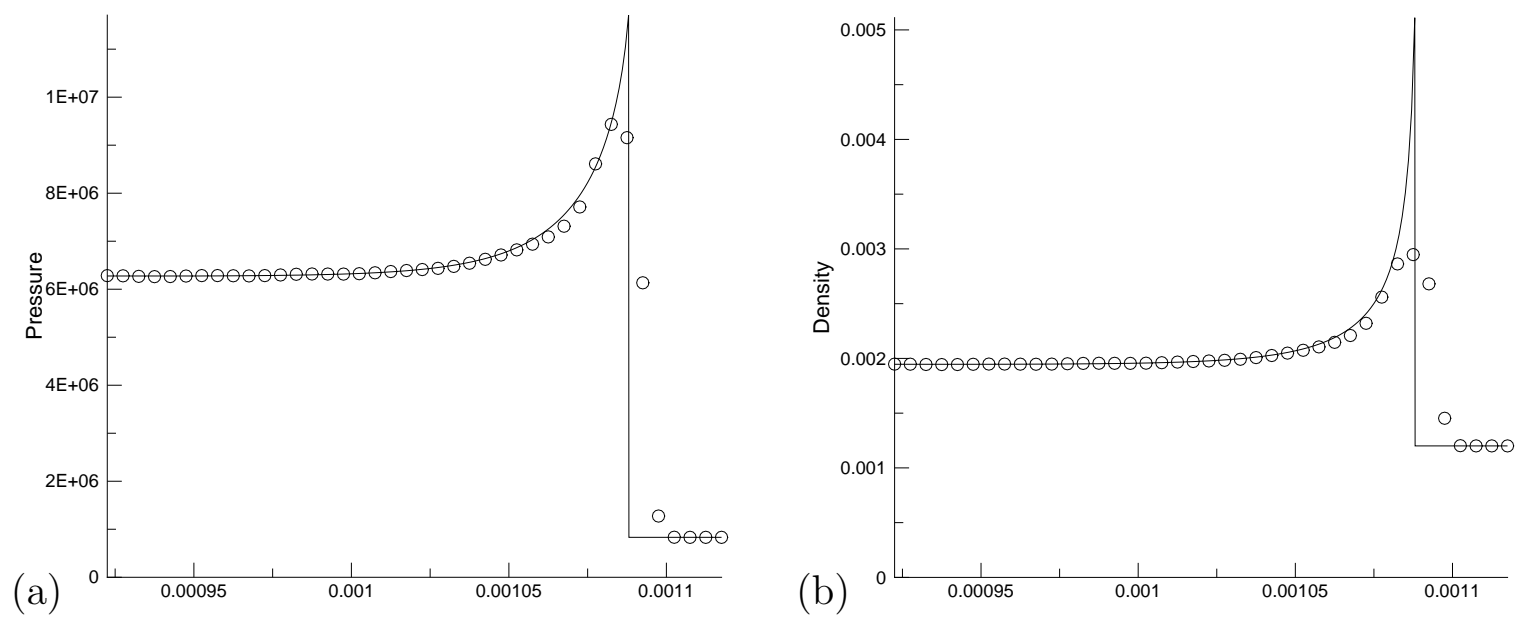

Figure 9. Pressure (a) and density (b) profiles at $t=10^{-8}$ calculated on the uniform mesh with $h=5 \cdot 10^{-6}$. Solid line is the ZND solution. 

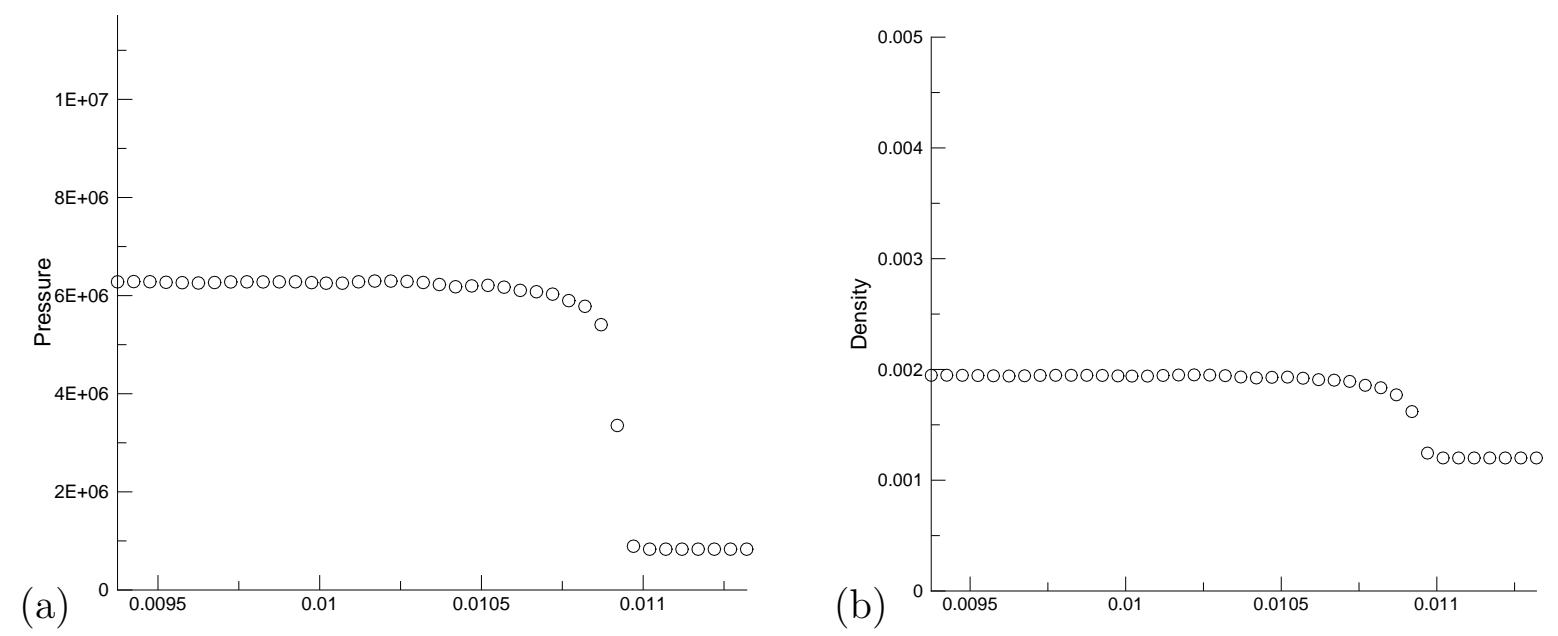

Figure 10. Pressure (a) and density (b) profiles at $t=10^{-7}$ calculated on the uniform mesh with $h=5 \cdot 10^{-5}$.
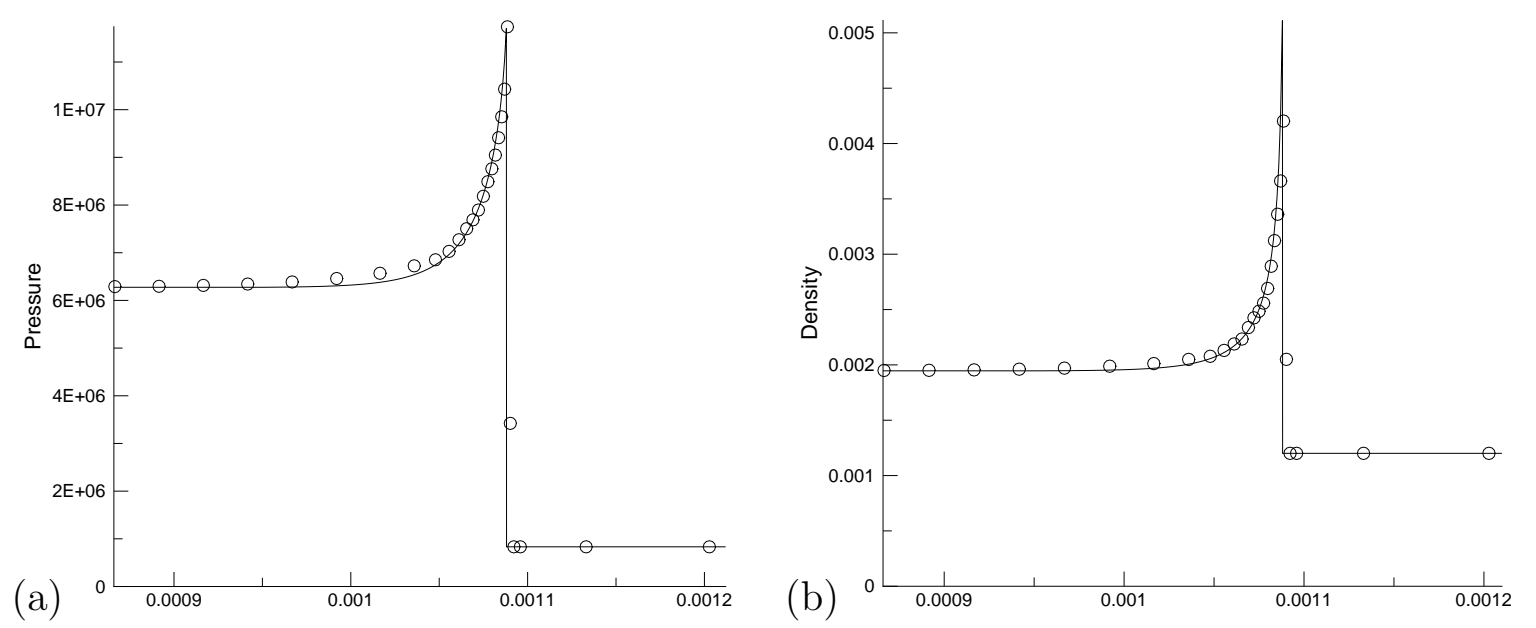

Figure 11. Pressure (a) and density (b) profiles at $t=10^{-8}$ calculated on the adapted mesh with initial spacing $h=5 \cdot 10^{-5}$ as in Fig. 10.

Next, we perform calculations on the adapted mesh. We start on the same coarse uniform mesh with $h=5 \cdot 10^{-5}$ and piece-wise initial data (34). After achieving the steady state, this solution and its induced adapted mesh are used as initial data for the adaptive modeling. Otherwise we would obtain an incorrect $D_{\mathrm{CJ}}$ velocity. The results are presented in Fig. 11 and Table 1. The mass fraction $Z$ is used as the control function $f$. Here at every time step we perform 20 mesh iterations, the coefficient of adaptation used is $c_{a}=0.7$, and the iterative parameter in (31) is $\tau=0.4$. It is seen from Fig. 11 that the spike profile is better resolved than that of in Fig. 9 since after the mesh adaptation the minimal spacing in the reaction zone is $h_{\min }=1.56 \cdot 10^{-6}$ which is about 3.2 times smaller than the uniform mesh spacing in Fig. 9. Thus, the spacing in the critical zone is decreased by a factor of 32 relative to the initial mesh. Note that the time step decreases approximately by a factor of 32 as well that follows from (14). In fact the effective reduction of $h$ is about 45 , because ahead the detonation wave (already over 2 cells) the spacing is $h \approx 7 \cdot 1 \cdot 10^{-5}$ and behind the burning zone $h \approx 2 \cdot 5 \cdot 10^{-5}$. Despite these facts the error to the adapted solution is slightly larger than that to the uniform mesh solution with $h=5 \cdot 10^{-6}$, as observed in Table 1 . 
This is due to the lack of nodes in the smooth part of the reaction zone. Nevertheless, due to adaptation, on the coarse mesh we obtained nearly the same accuracy as that of on the refined one. It is of interest to see that from the side of the heating shock the mesh is sharply condensed, within one cell the spacing is reduced by several tenths of times. In this manner the mesh is condensed for the non-reactive gas flow problems with shock waves [4]. From the side of the burnt gas, where the solution is smooth, the nodes concentrate gradually. For the smooth solution as $c_{a} \rightarrow \infty$ we get the optimal grid in the sense that the error in the norm $L_{\infty}$ on such a mesh is minimal [3].

Table 1

Numerical error for the pressure and density relative to ZND solution.

\begin{tabular}{|l|c|c|}
\hline & $\|\operatorname{Er}(p)\|_{L_{1}}$ & $\|\operatorname{Er}(\rho)\|_{L_{1}}$ \\
\hline Unifrom mesh, $h=5 \cdot 10^{-6}$ & $1.22 \cdot 10^{2}$ & $3.28 \cdot 10^{-8}$ \\
Adapted mesh, $h_{\min }=1.56 \cdot 10^{-6}$ & $1.37 \cdot 10^{2}$ & $4.00 \cdot 10^{-8}$ \\
\hline
\end{tabular}

\subsection{Unstable one-dimensional detonation}

First experiments and then a theoretical analysis, see [23,37], have shown that overdriven detonation may be unstable in a gas for some range of the parameters. Now we calculate one such a case of the unstable detonation for the Arrhenius kinetics model (4). The dimensionless parameters by reference to the uniform state ahead the detonation shock, moving to the right, are $\gamma=1.2, q_{o}=50, E^{+}=50$, the degree of overdrive $f=\left(D / D_{\mathrm{CJ}}\right)^{2}=1.6$, where $D$ is the shock speed, $K_{o}=230.75, R=1,(u, p, \rho, Z)_{\mathrm{unb}}=(0,1,1,1)$. This problem was also simulated numerically in $[25,8,44,42,29]$.

We conduct calculations in two ways: first, on the fixed mesh, and, second, with shock tracking on the moving mesh. In both cases the spacing $h$ is equal 0.05 , which corresponds to having 20 cells per half-reaction length for the ZND profile (20 pts $\left./ L_{1 / 2}\right)$, which is used as initial data. At the rear boundary we define the piston velocity as that of for the ZND profile, i.e. set $u=u_{\mathrm{bnt}}$, and the transmissive boundary conditions for $p, \rho, Z$. On the front boundary we define $(u, p, \rho, Z)_{\mathrm{unb}}$.

In the first case of the fixed mesh simulation we set $c_{\mathrm{cfl}}=0.5$. The modeling is performed in the laboratory frame. The shock pressure history is depicted in Fig. 12a. For comparison, the result obtained in [8] using the piecewise parabolic method (PPM) with shock fitting on the fixed mesh is also depicted. We observe a good agreement in the amplitude (the difference of peak pressure $\leq 3 \%$ ) and in the wave velocity.

Consider the length of the computational domain which we should retain behind the shock during modeling. By theory, see [58], the steady overcompressed detonation mode is possible if the formation of the rarefaction wave is not allowed after the burning process is ended. Therefore, one needs to move the piston with the medium velocity $u_{\text {bnt }}$ at point 

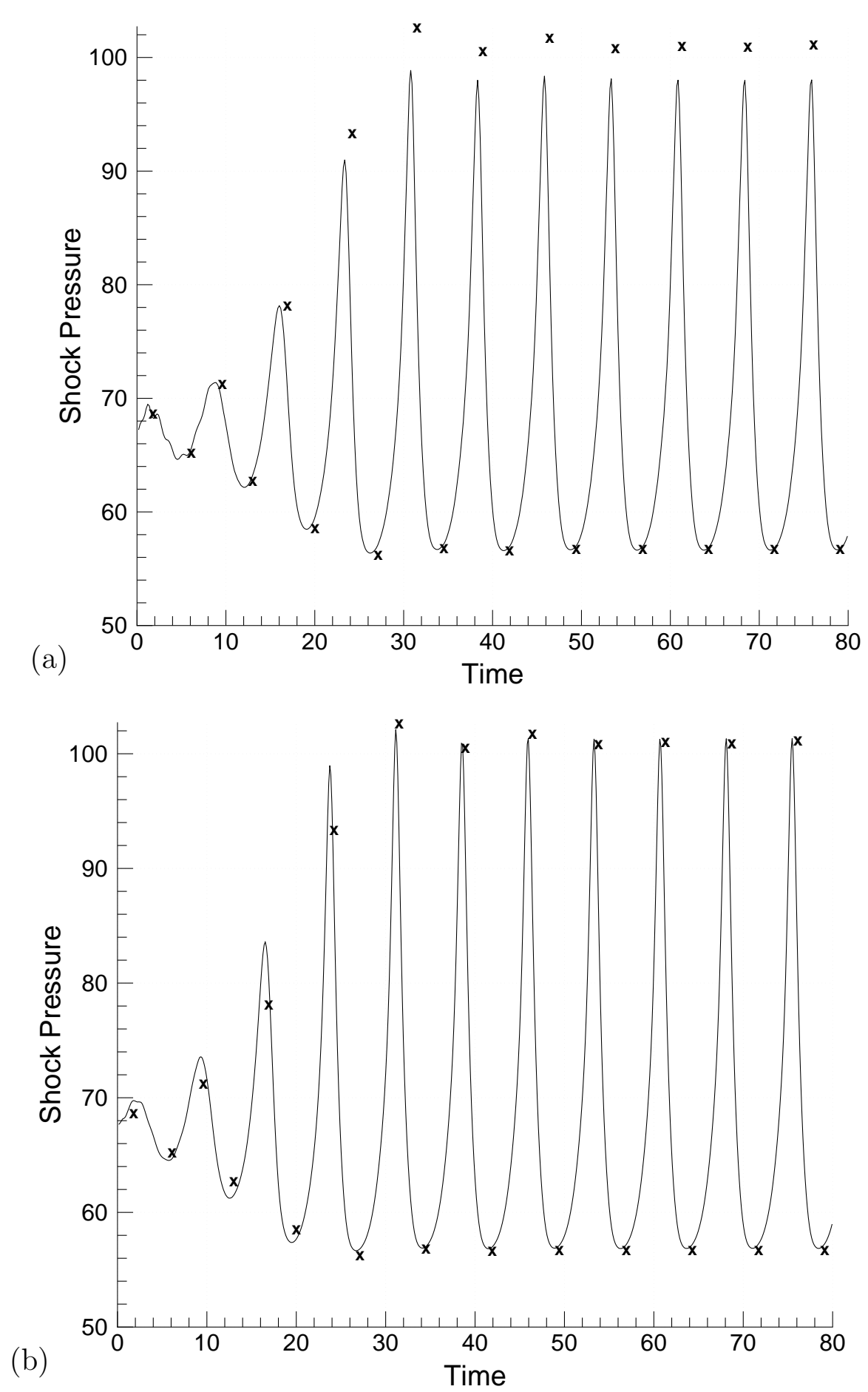

Figure 12. Peak pressure history for unsteady flow. Symbol "x" indicates the solution in [8], solid line is the present results. Calculation on the fixed mesh (a). Calculation with front tracking on the mesh moving with the shock speed $D(\mathrm{~b})$.

5 in Fig. 1 to support the overdriven detonation, which can not occur in the free mode without support. This value can be corrupted due to the disturbances moving from the flow domain to the burning zone left end with the speed of $u_{\mathrm{bnt}}-c_{\mathrm{bnt}}$, where $c_{\mathrm{bnt}}$ is the sound speed in the burnt gas. It is the left characteristic motion that defines the computational domain length. In addition, the zone right endpoint moves to the right with the shock 
velocity $D$ being not constant due to pulsations. Finally, the computational domain size $L_{\text {dom }}$ is estimated as

$$
L_{\mathrm{dom}}(t)=L_{\mathrm{dom}}(0)-\left(u_{\mathrm{bnt}}-c_{\mathrm{bnt}}\right) t+\int_{0}^{t} D(\tau) d \tau
$$

where $L_{\text {dom }}(0)$ is the initial zone length which is equal to 100 cells $\left(5 L_{1 / 2}\right)$. The value of the precursor shock speed $D$ periodically changes about the initial value of 8.613 due to pulsations from 7.9 to 10.5 . In reality the left characteristic moves to the right with the velocity $u_{\mathrm{bnt}}-c_{\mathrm{bnt}}=6.2489-4.2497=1.9992$. Integrating (35) numerically simultaneously with modeling we obtain $L_{\mathrm{dom}} \approx 534 L_{1 / 2}$ at $t=80$. When simulating we remove the left endcell when the characteristic $u_{\mathrm{bnt}}-c_{\mathrm{bnt}}$ crosses the right node of this cell and add one cell to the zone right end when the shock crosses the left node of this cell (because of shock smearing we keep several cells ahead the heating shock). The numerical experiments confirm the validity of (35). It is of interest that substituting the constant value $D_{o}=$ $D(0)$, taken from the ZND solution, in (35) gives nearly the same length $L_{\text {dom }}$ (with difference of several cells). Thus, with good approximation one can use the estimate

$$
L_{\mathrm{dom}}(t)=L_{\mathrm{dom}}(0)-\left(u_{\mathrm{bnt}}-c_{\mathrm{bnt}}\right) t+D_{o} t
$$

instead of using (35). If to calculate in the shock frame, i.e. define the initial and boundary velocity values as $u_{\text {shock }}=u_{\text {lab }}-D_{o}$, some high frequency instability in the solution arises.

In the second case we use front tracking and calculate on the uniform mesh, which moves to the right with the heating shock velocity $D$. The value of $D$ is determined using the Rankine-Hugoniot jump conditions. Actually, we obtain the shock velocity after solving the Riemann problem at the right endpoint $x_{I+1}^{n+1 / 2}$, where $D$ is the speed of the right shock in the wave pattern (likewise $d_{s h}$ is the speed of the left shock in Fig.3). At the predictor stage, to construct the preliminary grid at $t^{n+1}$, one uses the velocity $D$ determined at the preceding time level. The mesh moves to the right and when the left characteristic $u_{\mathrm{bnt}}-c_{\mathrm{bnt}}$ remains behind the 1st mesh node at the distance of $h$ we add one cell to the left. The estimate (35) is suitable to this case as well. We begin modeling with $c_{\mathrm{cfl}}=0.5$. However, after $t=45$ it is reduced down to 0.45 in order to escape overflow in computations. The shock pressure history is presented in Fig. 12b. We see that the results are the same as that in [8] where the fourth-order scheme (PPM) with front tracking is applied. Thus, this modeling confirms the idea stated in [1], namely the loss in accuracy occurs mainly in the shock smearing zone when a shock capturing method is used as in the case presented in Fig. 12a. If we apply front tracking, the numerical scheme in the subdomains of the smooth flow provides the same accuracy as the method of characteristics does. Modeling in the shock frame provides a bit less accuracy. Here the average value of the front peak pressure is $\approx 100$, meanwhile, in the laboratory frame calculation, depicted in Fig. 12b, it is $\approx 101$. 
It may be of interest to note that the admissible time step $\Delta t$ in the moving mesh calculation is substantially larger than that of on the fixed mesh. In fact, if we perform estimation of $\Delta t$ at $t=0$, from (14) in the left endcell, where

$$
d_{1}^{\mathrm{rght}}=u_{\mathrm{bnt}}+c_{\mathrm{bnt}}=10.499, \quad d_{2}^{\mathrm{lft}}=u_{\mathrm{bnt}}-c_{\mathrm{bnt}}=1.9992, \quad w_{1}=w_{2}=D=8.613
$$

we obtain

$$
\Delta t_{3 / 2}=\frac{h}{\max \left(d_{1}^{\mathrm{rght}}-w_{2},-d_{2}^{\mathrm{lft}}+w_{1}\right)}=7.55 \cdot 10^{-3} .
$$

Meanwhile on the fixed mesh, where $w_{i}=0$, we have $\Delta t_{3 / 2}=4.76 \cdot 10^{-3}$.

In the right $I$ th cell, immediately behind the shock, with the values obtained from the Riemann problem on the moving mesh $d_{I}^{\mathrm{rght}}=10.626$ and $d_{I+1}^{\mathrm{lft}}=4.782$, we have $\Delta t_{I+1 / 2}=1.31 \cdot 10^{-2}$. On the fixed mesh with $d_{I}^{\mathrm{rght}}=10.635, d_{I+1}^{\mathrm{ft}}=4.76$ the time step is $\Delta t_{I+1 / 2}=4.70 \cdot 10^{-3}$. Thus, the time steps ratio grows from 1.59 in the left endcell to 2.78 in the right endcell. This is due to the left characteristic moves in the same direction as the grid nodes on the moving mesh. Indeed, by (13) we should use the minimal step and multiply it by $c_{\text {cfl }}$. Finally, by $t=80$ we perform 35597 time steps on the fixed mesh and 23488 steps on the moving mesh.

\subsection{Unstable two-dimensional detonation}

As in one-dimensional case the planar flow is also unstable in a range of the flow parameters, e.g. see $[48,49]$. The theory of this phenomena, giving rather qualitative sketch, can be found e.g. in [26]. The structure of the two-dimensional flow is rather complicated. Thus, a precise numerical simulation is of interest.

We present the results of modeling the unstable detonation in a channel drawn in $[9,44]$, when the transversed waves (or cellular structure) are produced. The gas with degree of overdrive $f=1.2$ flows in the channel with the height equal $10 L_{1 / 2}$. We use the kinetics model (4) with dimensionless parameters $\gamma=1.2, q_{o}=50, E^{+}=10, K_{o}=3.124$, $(u, v, p, \rho, Z)_{\mathrm{unb}}=(0,0,1,1,1)$. The ZND profile is used as initial data. On the top and bottom of the channel the periodic boundary conditions are applied. On the rear boundary we define the piston velocity $u=u_{\text {bnt }}$, and transmissive boundary conditions for the other parameters. The rectangular mesh is $h_{x}=h_{y}=h=0.05$, i.e. $20 \mathrm{pts} / L_{1 / 2}$. The planar detonation front is disturbed allowing it within the first $10^{3}$ time steps to ingest a small region of fluid ( 5 cells in width and 100 in height) with the rate constant $K_{o}^{\prime}=0.8 K_{o}$.

The question of interest is how far should we place the rear boundary, i.e. piston. The fact is that we perform calculations up to time $t \approx 62$ and, therefore, with the steady ZND's mode values $D_{o}=7.459, u_{\mathrm{bnt}}=4.704, c_{\mathrm{bnt}}=4.0$ and initial $L_{\mathrm{dom}}(0)=5 L_{1 / 2}$ via $(36)$ 
one estimates the length of $424 L_{1 / 2}$ or 8480 cells to be used in the end. It is not realistic to calculate on such a mesh. To overcome this difficulty, we performed the following numerical experiment. First, it was conducted three calculations on the rough mesh with $h=0.2$ till time $t=40$ when the flow structure is developed. In the first case we calculate throghout the domain of $L_{\mathrm{dom}} \approx 275 L_{1 / 2}$ (1375 cells) determined via (36). In the second case we set $L_{\mathrm{dom}}=60 L_{1 / 2}(300$ cells $)$, and in the third case $L_{\mathrm{dom}}=40 L_{1 / 2}$ (200 cells $)$. By the reason stated in section 5.2 we calculate in the laboratory frame and keep eliminating cells at the left end and adding cells ahead the shock front. The difference of the maximal peak pressures for the 1 st and 2 nd cases at $t=40$ is less than $0.01 \%$, and for the 1 st and 3rd cases is less than $1 \%$. We believe that for our calculations on the refined mesh it is sufficient to keep the rear boundary at the distance of $40 L_{1 / 2}$ behind the detonation wave front. Thus, the problem is computed on the $800 \times 200$ mesh. In all calculations the CFL number is $c_{\mathrm{cfl}}=0.5$.

First, the calculation is executed on the rectangular mesh. For $t=60$ the running time on $\mathrm{PC}$ Pentium $2.0 \mathrm{GHz}$ is about 36 hours. Fig. 13 shows the pressure contours from $t=60.060$ to 62.735 (see also Figs. 17a, 19a). The general structure of the solution is similar to that in $[9,44]$.

The moving mesh calculation is executed in two ways. In the first, the front tracking procedure, described in sec. 3.4, is employed. Here the right edges of the right row cells are the precursor shock front. At every time step, after the wave front has been shifted, the functional (32) is employed to redistribute the boundary nodes and (27) to move the internal nodes. In addition we can switch on the adaptive procedure, see examples of the meshes in Fig. 14. In the second case we apply the global adaptation without front tracking, see the meshes in Figs. 16,18.

When adapting, as a control function $f$ it is used the density, see mesh in Fig. 14a, and pressure, Figs. 14b,16,18. The choice of $f$ is defined by what traits of the flow pattern to be investigated in detail. The parameter $D_{\max }$ in (30) is set from 10 to 15 . At every time step we perform 2 mesh iterations (therefore, update the gas dynamics parameters three times), and set $\tau=0.9$ in (29), coefficient $c_{a}$ is set from 0.07 to 0.1 . When adapting, at first mesh iteration the initial mesh at $t^{n+1}$ is determined by the formulas

$$
x_{i, j}^{n+1}=x_{i, j}^{n}+D_{o} \triangle t, \quad y_{i, j}^{n+1}=y_{i, j}^{n} .
$$

Using adaptation we aim to resolve as much as possible the main (i.e. most intensive) singularities in the solution and by this reason we condense strongly the grid lines. Admissible time step $\Delta t$ falls from $1.5 \cdot 10^{-3}$ on the rectangular mesh by 5 to 10 orders of magnitude, i.e. practically goes to zero. Note that $\Delta t$ is not a constant even for the steady problems, the mesh constantly "breaths", and, accordingly, the value of $\Delta t$ periodically decreases and then increases, etc. Indeed, we can not calculate within a long period of the "flow" time with such an infinitesimal $\Delta t$. Thus, we can operate in two ways: first, 
starting the adaptation when necessary from the initial state, obtained on the rectangular mesh, or, second, weakening adaptation by reducing the coefficient of adaptation $c_{a}$ and, therefore, increasing $\Delta t$.

Further we will be concern with some particular features of the flow pattern and compare the results of the fixed and moving mesh calculations. The density plots for the rectangular mesh modeling at $t=60.44$ are depicted in Fig. 15a. The flow structure behind the Mach stem, inside the cell, is rather unclear due to the presence of oscillations. We can not observe neither how close the fire zone approaches the precursor shock nor the slip lines emanating from the upper and lower triple points. Results of front tracking, see Fig. 15b, show the front part of the fire zone and slip lines impinging on it. It is of interest that the form and location of the fire zone differs from qualitative results predicted in [26]. In [26], the fire zone lays immediately behind the Mach stem and is parallel, meanwhile in Fig. 15b it bends strongly. Evidence that it is the fire zone one can find in Fig. 15d with the mass fraction plots depicted. The fire zone has no a structure behind the incident shock. Among all flow parameters the density plots have the most complicated structure, which can be better seen in Fig. 15c of the adaptive mesh calculation. Indeed, we can not resolve all the solution traits by grid lines condensing, but the main discontinuities are reflected in the mesh structure rather well, see Fig. 14a. Meanwhile the density "feels" the fire zone and a bit slip lines, see Fig. 14a, using the pressure as $f$ gives a better resolution of the transverse waves, both intensive and weak, emanating from the triple points and beginning to emerge behind the incident shock, see the meshes in Figs.14b,16,18. Capturing the slip lines by grid lines condensing is not subject to an instability, as may be expected. As shown in [4] for the example of the contact discontinuity in the one-dimensional linear model, our adaptive method can not prevent slip lines smearing with time. Besides, the "barrier" property of this grid generator prevents the mesh cells from infinitesimal refinement $[14,4]$, which is regulated by the coefficient $c_{a}$ value.

Between two transverse shocks, depicted in Fig. 16b (one intensive and the other weak), the grid cell size changes sharply in the normal direction to those shocks. It seems possible that the approximation on such a mesh is to deteriorate. However, first, as shown in [4], a large ratio of the neighboring cells size (one in the smeared-out shock zone and the other in the domain of smooth flow), does not deteriorate the solution. Second, the narrow cells in the shock zone lead the scheme, based on the integral conservation laws, to update the flow parameters nearly as the shock fitting procedure via the Rankine-Hugoniot jump conditions [4]. This conclusion can be drawn from the following consideration. If to draw a closed contour along the shock, one side to the left of the discontinuity and the other to the right, and to direct the contour width to zero, from the system of conservation laws, written in the integral form, in the limit we get the Rankine-Hugoniot jump conditions [46,31]. The imaginarily drawn line, passing through the centers of narrow cells in the shock zone, looks like that contour when the width-to-length cell ratio tends to zero. Thus, due to adaptation, the flow solver, by grid cell size variation, is adjusted to perform the habitual calculations in the domains of smooth flow and to operate as the 
front fitting procedure in the shock zone. The experimental evidence of this statement can be seen by comparing the pressure contours obtained on the rectangular and adapted meshes. Oscillations in pressure behind the precursor shock, observed in Fig. 17a, disappear in Fig. 17d. In this regard the global adaptation, Fig. 17d, works like front tracking, Fig. 17b,c. Note that with front tracking one can only treat the precursor shock (and it is a special procedure), meanwhile the global adaptation operates with the "intensive singularities" throughout the flow automatically.

The fragment of the mesh near the top boundary is shown in Fig. 16c. If the constrained minimization is not used to the boundary nodes redistribution, we can not obtain such a fine-mesh structure. Numerical experiments have shown that instability in the adaptive mesh generation on the boundary (if no constrained minimization) leads to interior domain instability, rather far from the boundary.

\section{Concluding remarks}

In this work, the unsplit scheme to model the reactive gas flow on the moving meshes has been presented. This scheme allows for calculations coupled with front tracking, which enhance the accuracy of simulation. Using the moving adaptive meshes allows us to concentrate locally the grid points in the vicinity of singularities: shocks, slip lines, rarefaction waves, and fire zones, while keeping the simple mesh structure and invariable number of nodes. Numerical experiments have shown that using adaptive meshes allows efficiently resolving the thin structures of the solution. Strongly condensed grid lines in the vicinity of shocks lead the flow solver is adjusted to perform calculations like using the jump conditions and this, in turn, gives significant accuracy enhancement of the modeling.

In the 3D calculations, some principal difficulties in mesh generation still need to be overcome. First, it concerns with the search of a transformation providing a homeomorphic mapping of the unit cube onto an arbitrary domain in the continuous approach. Note that in the $3 \mathrm{D}$ case it is unknown yet whether a harmonic mapping of an arbitrary domain onto a convex domain (unit cube) with a given one-to-one mapping between the boundaries is always a homeomorphism. There is an example (see [24]), when, subject to some conditions imposed on the boundaries, the harmonic mapping is not a homeomorphism. Second, for a hexahedral cell there unknown a finite number of conditions using which one can definitely say whether this cell is invertible or not [54]. One of possible ways to overcome these difficulties is to seek directly a discrete mapping. One such example was considered in [34]. Anyway, an additional thorough study is required to execute in this direction. 


\section{Acknowledgments}

The work of B.N. Azarenok was partially supported by Russian Fund of Fundamental Research (project code 02-01-00236). The work of T. Tang was partially supported by Hong Kong Research Grant Council (Project Code HKBU2045/02P and HKBU 2018/03P) and International Research Team on Complex System, Chinese Academy of Sciences.

\section{References}

[1] G.B. Alalykin, S.K. Godunov (Ed.), I.L. Kireeva, and L.A. Pliner, Solution of OneDimensional Problems in Gas Dynamics on Moving Meshes, Nauka Press (Moscow, 1970).

[2] B.N. Azarenok, Realization of a second-order Godunov's scheme, Comp. Meth. in Appl. Mech. and Engin., 189 (2000), pp. 1031-1052.

[3] B.N. Azarenok and S.A. Ivanenko, Application of moving adaptive grids for numerical solution of nonstationary problems in gas dynamics, Int. J. for Numer. Meth. in Fluids, 39 (2002), No. 1, pp. 1-22.

[4] B.N. Azarenok, Variational barrier method of adaptive grid generation in hyperbolic problems of gas dynamics. SIAM J. Numer. Anal., 40 (2002), No. 2, pp. 651-682.

[5] B.N. Azarenok, S.A. Ivanenko, and T. Tang, Adaptive mesh redistribution method based on Godunov's scheme, Comm. Math. Sci., 1 (2003), No. 1, pp. 152-179.

[6] W. Bao and S. Jin, The random projection method for hyperbolic systems with stiff reaction terms, J. Comput. Phys., 163 (2000), pp. 216-248.

[7] M. Ben-Artzi, The generalized Riemann problem for reactive flows, J. Comput. Phys., 81 (1989), pp. $70-101$.

[8] A. Bourlioux, A.J. Majda, and V. Roytburd, Theoretical and numerical structure for unstable one-dimensional detonations, SIAM J. Appl. Math., 51, 1991, No. 2, pp. 303343.

[9] A. Bourlioux, A.J. Majda, Theoretical and numerical structure for unstable twodimensional detonations, Combustion and Flame, 90 (1992), pp. 211-229.

[10] J.U. Brackbill and J.S. Saltzman, Adaptive zoning for singular problems in two dimensions, J. Comput. Phys., 46 (1982), pp. 342-368.

[11] W.M. Cao, W.Z. Huang, and R.D. Russel, An $r$-adaptive finite element method based upon moving mesh PDEs, J. Comput. Phys. 149 (1999), pp. 221-244.

[12] J. Casper and M.H. Carpenter, Computational considerations for the simulation of shockinduced sound, SIAM J. Sci. Comp., 19 (1998), No. 3, pp. 813-828. 
[13] A.A. Charakhch'yan and S.A. Ivanenko, Curvilinear grids of convex quadrilaterals, USSR Comput. Math. Math. Phys. 28 (1988), No. 2, pp. 126-133.

[14] A.A. Charakhch'yan and S.A. Ivanenko, A variational form of the Winslow grid generator, J. Comput. Phys., 136 (1997), pp. 385-398.

[15] V.A. Cherkashin, A.V. Zabrodin, Method of numerical modeling the detonation wave, Keldysh Institute of Applied Mathematics, USSR Academy of Sciences, Internal report (1974).

[16] A.J. Chorin, Random choice solution of hyperbolic systems, J. Comput. Phys., 22 (1976), pp. $517-531$.

[17] P. Colella, A. Majda, and V. Roytburd, Theoretical and numerical structure for reacting shock waves, SIAM J. Sci. Statist. Comput., 7 (1986), pp. 1059-1080.

[18] R. Courant and K.O. Friedrichs, Supersonic Flow and Shock Waves, Springer-Verlag (1985).

[19] M. G. Crandall and A. Majda, The method of fractional steps for conservation laws, Numer. Math., 34 (1980), pp. 285-314.

[20] Yu.N. Deryugin, V.P. Kopyshev, and B.P. Tikhomirov, On detonation calculation by Forest Fire model using Godunov's method, Questions of Atomic Science and Technique, 1 (1990), pp. $48-52$.

[21] A.S. Dvinsky, Adaptive grid generation from harmonic maps on Riemannian manifolds, $J$. Comput. Phys., 95 (1991), pp. 450-476.

[22] B. Engquist and B. Sjögreen, The convergence rate of finite difference schemes in the presence of shocks, SIAM J. Numer. Anal., 35 (1998), No. 6, pp. 2464-2485.

[23] J.J. Erpenbeck, Stability of idealized one-reaction detonations, Phys. Fluids, 7 (1964), pp. 684-696.

[24] F.T. Farrell and L.E. Jones, Some non-homeomorphic harmonic homotopy equivalences. Bull. London Math. Soc., 28, 1996, pp. 177-182.

[25] W. Fickett and W.W. Wood, Flow calculations for pulsating one-dimensional detinations, Phys. Fluids, 9 (1966), pp. 903-916.

[26] W. Fickett and W.C. Davis, Detonation, University of California Press, (Berkeley, CA, 1979).

[27] V.E. Fortov, B. Goel, C.D. Munz, A.L. Ni, A.V. Shutov, and O.Yu. Vorobiev, Numerical simulation of Nonstationary Fronts and Interfaces by the Godunov Method in Moving Grids, Nuclear Science and Engineering, 123 (1996), pp. 169-189.

[28] C. Helzel, R.J. LeVeque, and G. Warneke, A modified fractional step method for the accurate approximation of detonation waves, SIAM J. Sci. Comput., 22, No. 4 (2000), pp. $1489-1510$. 
[29] P. Hwang, R. Fedkiw, B. Merriman, A.R. Karagozian, and S.J. Osher, Numerical resolution of pulsating detonation waves, Combustion Theory and Modeling, 4, (2000), No. 3, pp. $217-$ 240 .

[30] S.K. Godunov and G.P. Prokopov, On computation of conformal transformations and construction of difference meshes, Zh. Vychisl. Mat. Mat. Fiz., 7 (1967), pp. 1031-1059.

[31] S.K. Godunov (Ed.), A.V. Zabrodin, M.Ya. Ivanov, A.N. Kraiko, and G.P. Prokopov, Numerical Solution of Multi-Dimensional Problems in Gas Dynamics, Nauka Press (Moscow, 1976); (French translation: Résolution Numérique des Problémes Multidimensionnels de la Dynamique des Gaz, Mir, Moscou, 1979.)

[32] S.K. Godunov, Equations of Mathematical Physics, Nauka Press (Moscow, 1979).

[33] S.K. Godunov, Reminiscenses about difference schemes, J. Comput.Phys., 153 (1999), pp. $6-25$.

[34] Ivanenko S.A. Harmonic Mappings, Chapt. 8 in Handbook of Grid Generation. CRC Press, Boca Raton, Fl, 1999.

[35] O.B. Khairullina, A.F. Sidorov, O.V. Ushakova, Variational methods of Construction of optimal grids, Chapt. 36 in: Handbook of Grid Generation, J.F. Thompson et al. eds., CRC Press, Fl, (1999).

[36] A.G. Kulikovskii, N.V. Pogorelov, and A.Yu. Semenov, Mathematical Aspects of Numerical Solution of Hyperbolic Systems, Monographs and Surveys in Pure and Applied Mathematics, 188, (Chapman and Hall/CRC, Boca Raton, FL, 2001).

[37] H.I. Lee and D.S. Stewart, Calculation of linear detonation instability: one-dimensional instability of plane detonation, Journal of Fluid Mechanics, 216 (1990), pp. 103-132.

[38] B. van Leer, Towards the ultimate conservative difference scheme. V. A second order sequel to Godunov's methods, J. Comput.Phys., 32 (1979), pp. 101-136.

[39] V.D. Liseikin, On generation of regular grids on $n$-dimensional surfaces, USSR Comput. Maths. Math. Phys., 31 (1991), No. 11, pp. 47-57.

[40] V.V. Ostapenko, Convergence of Finite-Difference Schemes behind a Shock Front, Comput. Math. Math. Phys., 37 (1997), No. 10, pp. 1161-1172.

[41] E. Oran and J.P. Boris, Numerical Simulation of Reactive Flow, Elsevier, (New York, 1987).

[42] M.V. Papalexandris, A. Leonard, and P.E. Dimotakis, Unsplit schemes for hyperbolic conservation laws with source terms in one space dimension, J. Comput.Phys., 134 (1997), pp. $31-61$.

[43] R.B. Pember, Numerical methods for hyperbolic conservation laws with stiff relaxation I. Spurious solutions, SIAM J. Appl. Math., 53 (1993), pp. 1293-1330.

[44] J.J. Quirk, Godunov-type schemes applied to detonation flows, Combustion in High-Speed Flows, ed. J. Buckmaster et al, Dordrecht: Kluwer, (1994), pp. 575-596. 
[45] A.V. Rodionov, Increase of accuracy of Godunov's scheme, USSR Computational Math. and Math. Phys., 27, No. 12 (1987), pp. 1853-1860.

[46] B.L. Rozhdestvenskii and N.N. Yanenko, Systems of Quasilinear Equations and Their Applications to Gas Dynamics, Amer. Math. Soc. Transl. of Math. Monographs, 55, AMS, Providence, RI, 1983.

[47] R. Schoen and S. T. Yau, On univalent harmonic maps between surfaces, Invent. Math., 44 (1978), pp. 265-278.

[48] G.J. Sharpe, Linear stability of idealized detonations, Proc. of Royal Soc. of Lond., Ser. A, 453 (1997), pp. 2603-2625.

[49] M. Short and D.S. Stewart, Cellular detonation stability, Part 1. A normal-mode linear analysis, Journal of Fluid Mechanics, 368 (1998), pp. 229-262.

[50] H.Z. Tang ang T. Tang, Adaptive mesh methods for one- and two-dimensional hyperbolic conservation laws, SIAM J. Numer. Anal., 41 (2003), pp. 487-515.

[51] T. Tang and Z.-H. Teng, Error bounds for fractional step methods for conservation laws with source terms, SIAM J. Numer. Anal., 32 (1995), pp. 110-127.

[52] T. Tang, Convergence analysis for operator splitting methods to conservation laws with stiff source terms, SIAM J. Numer. Anal., 35 (1998), pp. 1939-1968.

[53] V.T. Ton, Improved shock-capturing methods for multicomponent and reacting flows, $J$. Comput. Phys., 128 (1996), pp. 237-253.

[54] Ushakova O.V. Conditions of nondegeneracy of three-dimensional cells. A formula of a volume of cells, SIAM J. Sci. Comp, 23 (2001), No. 4, pp. 1273-1289.

[55] N.N. Yanenko, N.T. Danaev, and V.D. Liseikin, On variational method to cunstructing meshes, Chislen. Metody Mekhan. Sploshn. Sredy, 8, No. 4 (1977), Novosibirsk, pp. 157163.

[56] H. Weyl, Shock waves in arbitrary fluids, Comm. Pure Appl. Math., 2, No. 2-3 (1949), pp. 103-122.

[57] A. Winslow, Numerical solution of the quasi-linear Poisson equation in a nonuniform triangle mesh, J. Comput. Phys., 1 (1966), pp. 149-172.

[58] Ya.B. Zeldovich and S.A.Kompaneets, Theoriya Detonatsii (Theory of Detonations), Gostekhizdat (Moscow, 1955) (English translation: Academic Press, New York, 1960.) 
(a)

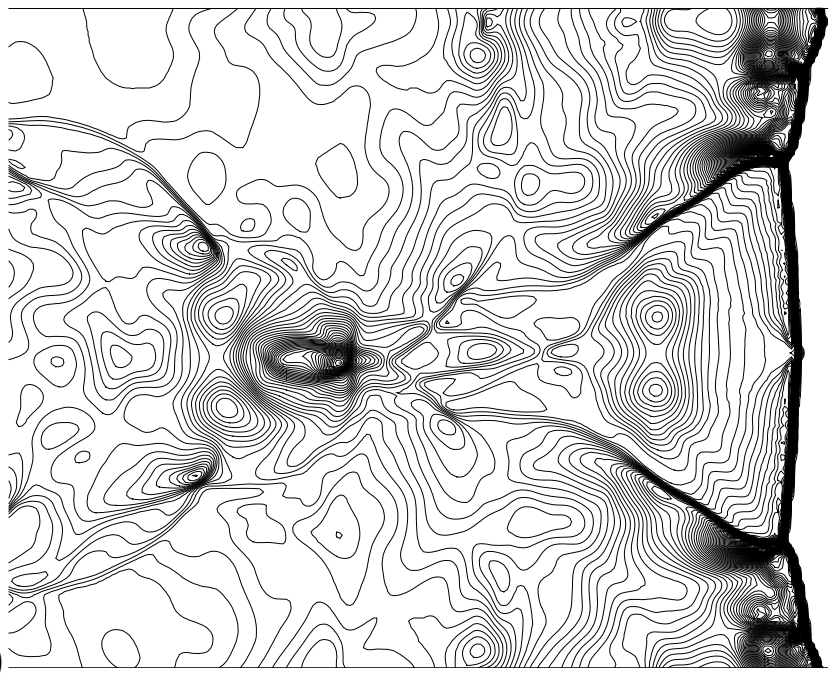

(b)
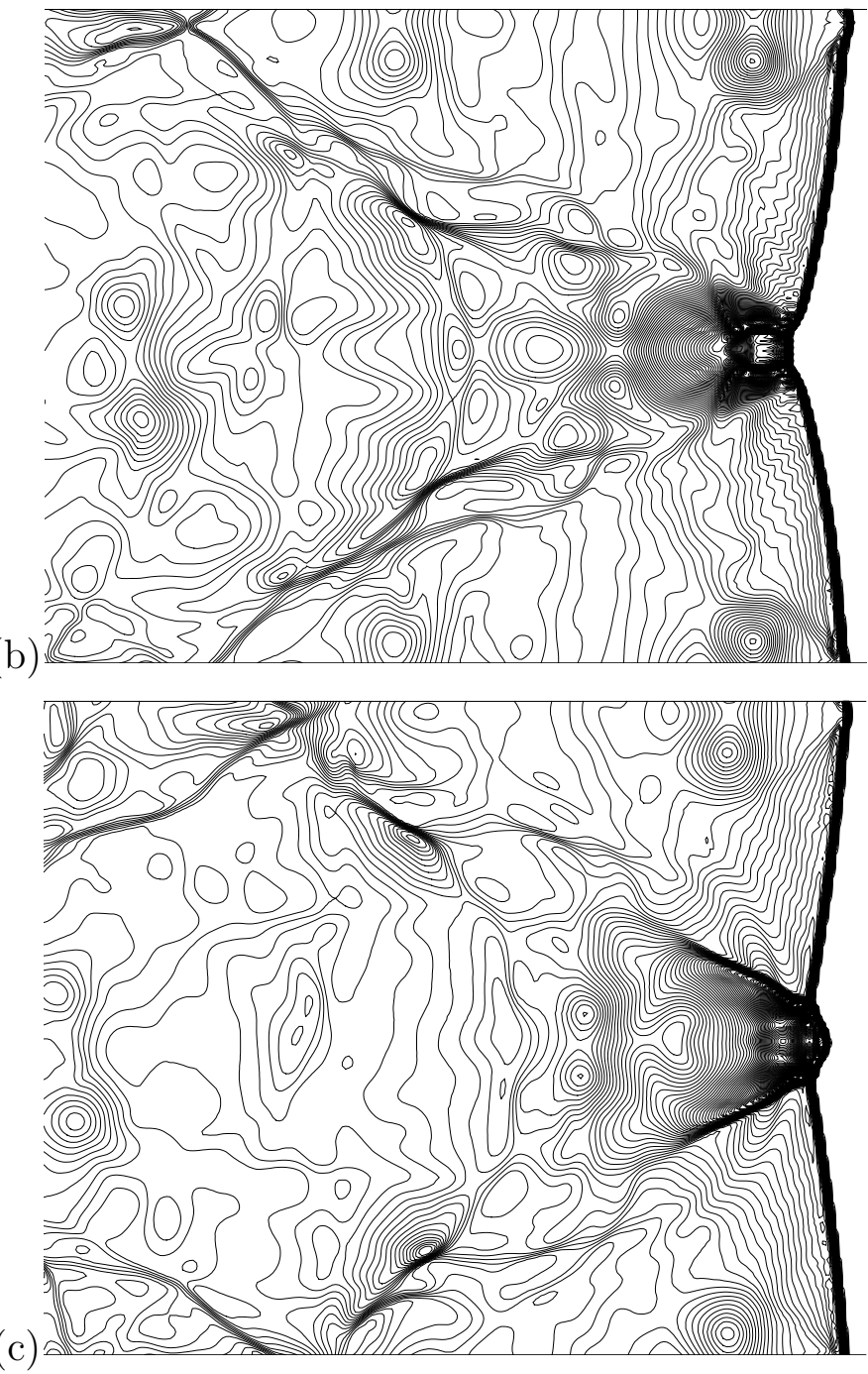

(d)

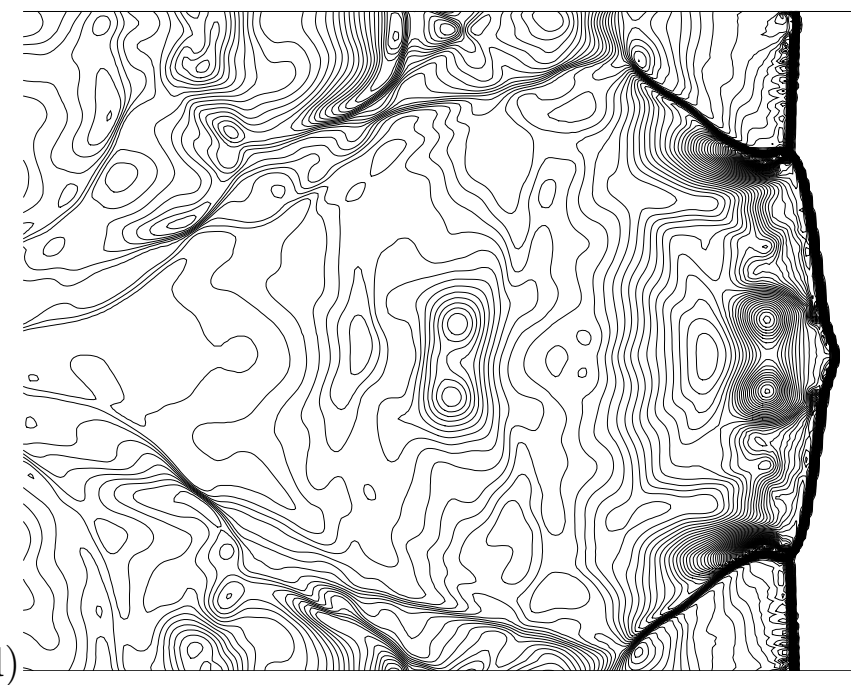

(e)

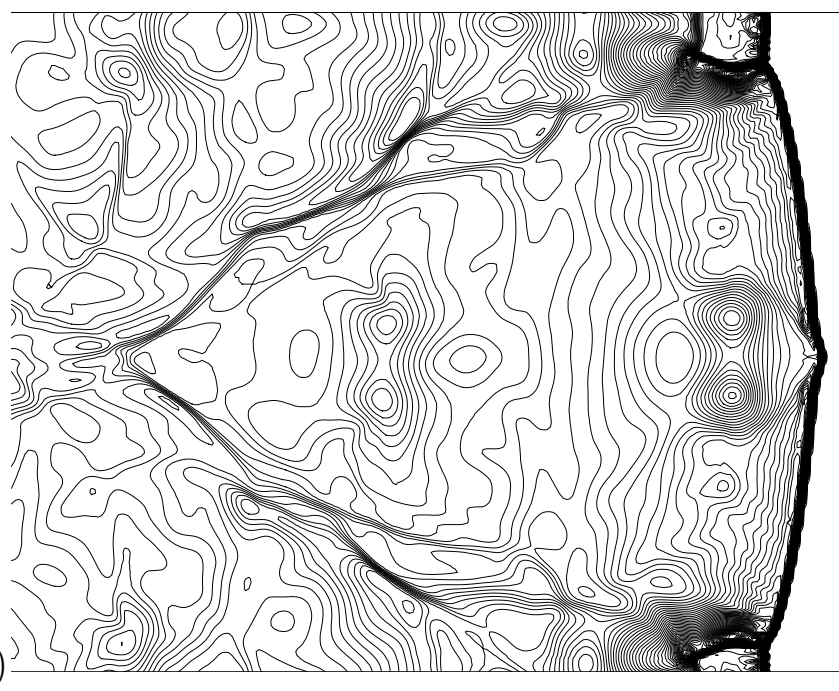

(f)

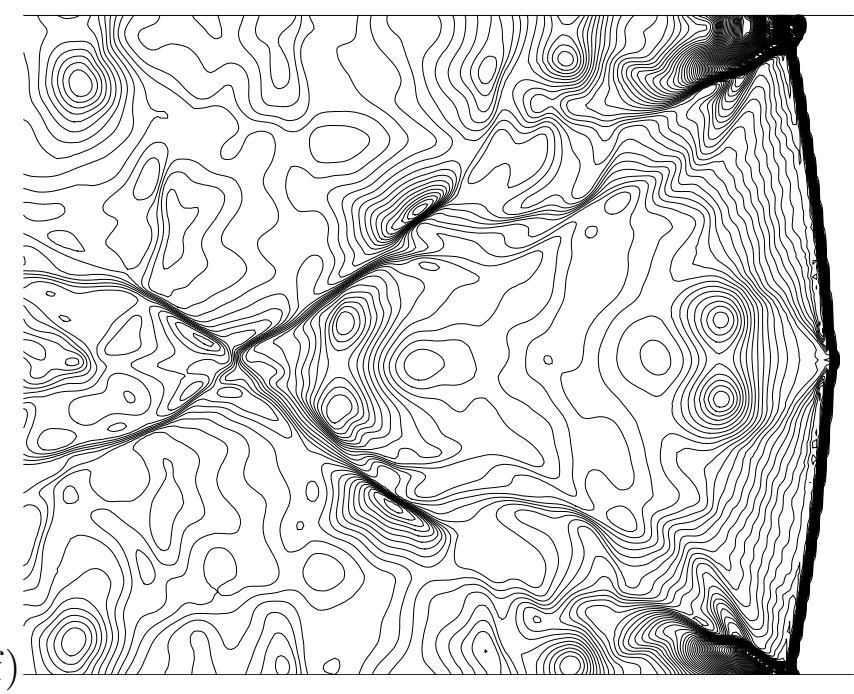

Figure 13. Pressure contours at $t=60.060$ (a), 60.824 (b), 61.205 (c), 61.981 (d), 62.362 (e), 62.735 (f) for rectangular $800 \times 200$ mesh calculation. Only 250 cells in the $x$-direction near the wave front are presented. 


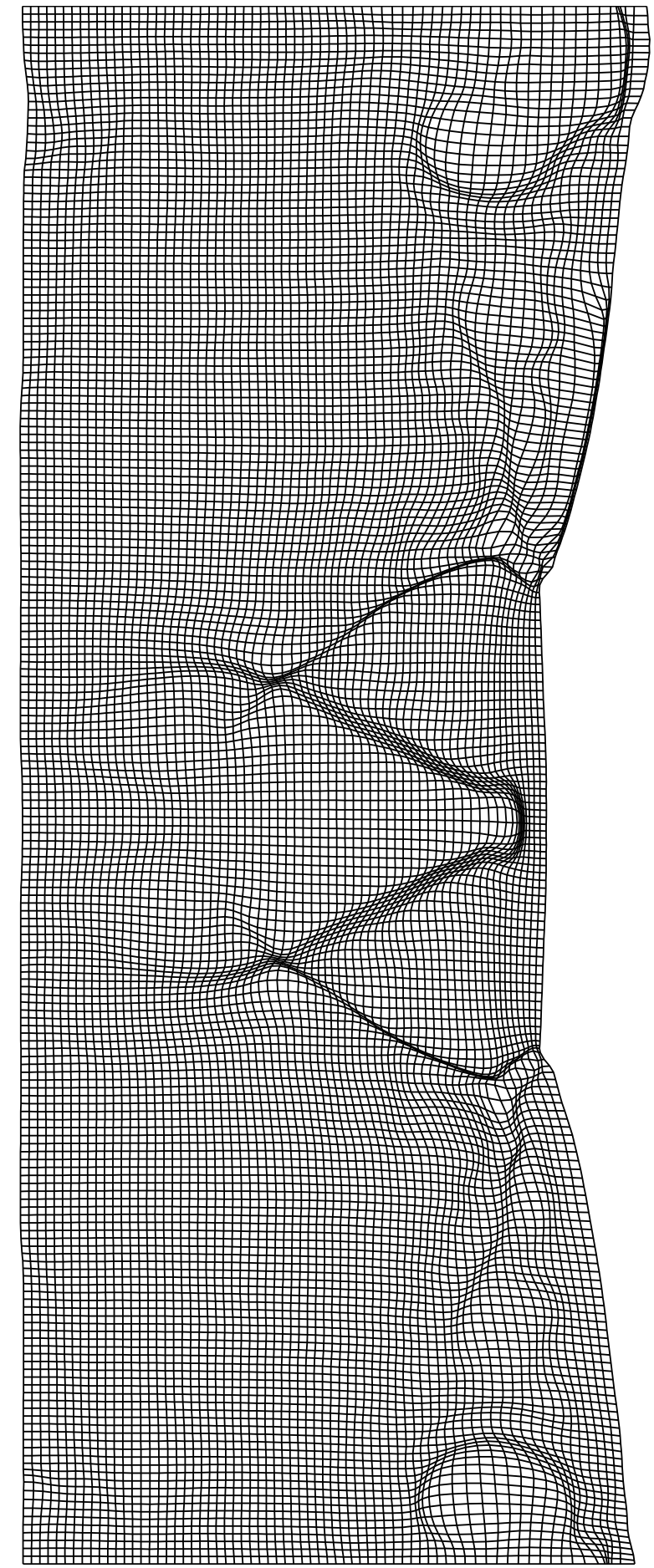

(a)

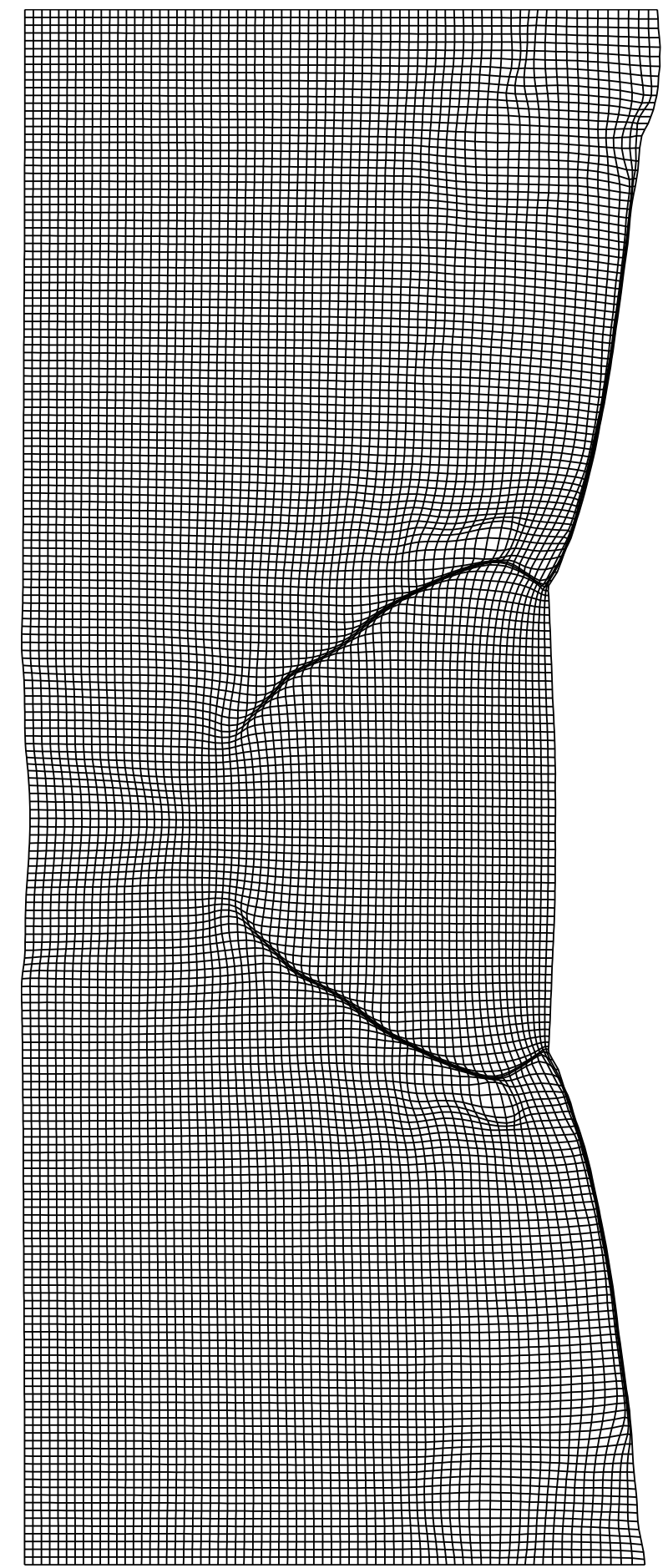

(b)

Figure 14. Front tracking and adaptation. Meshes at $t=60.44$ with density (a) or pressure (b) as a control function $f$. Only 60 front cells in the $x$-direction are depicted. 


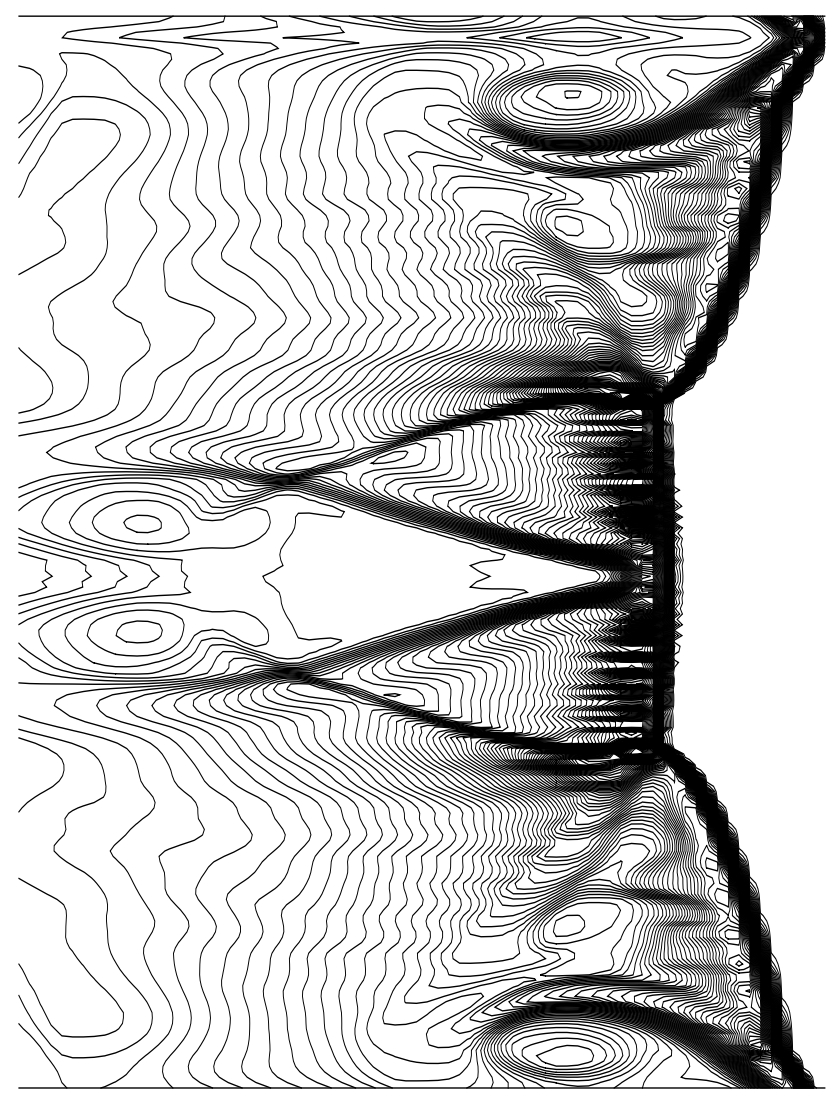

(a)

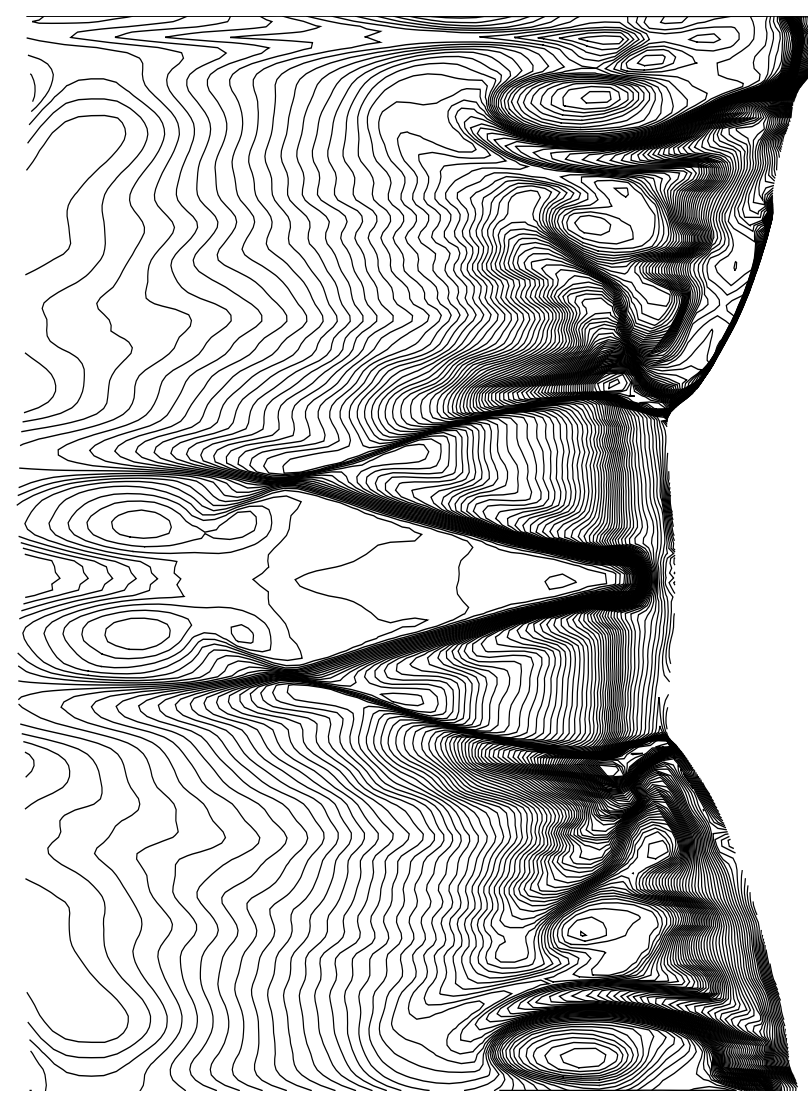

(c)

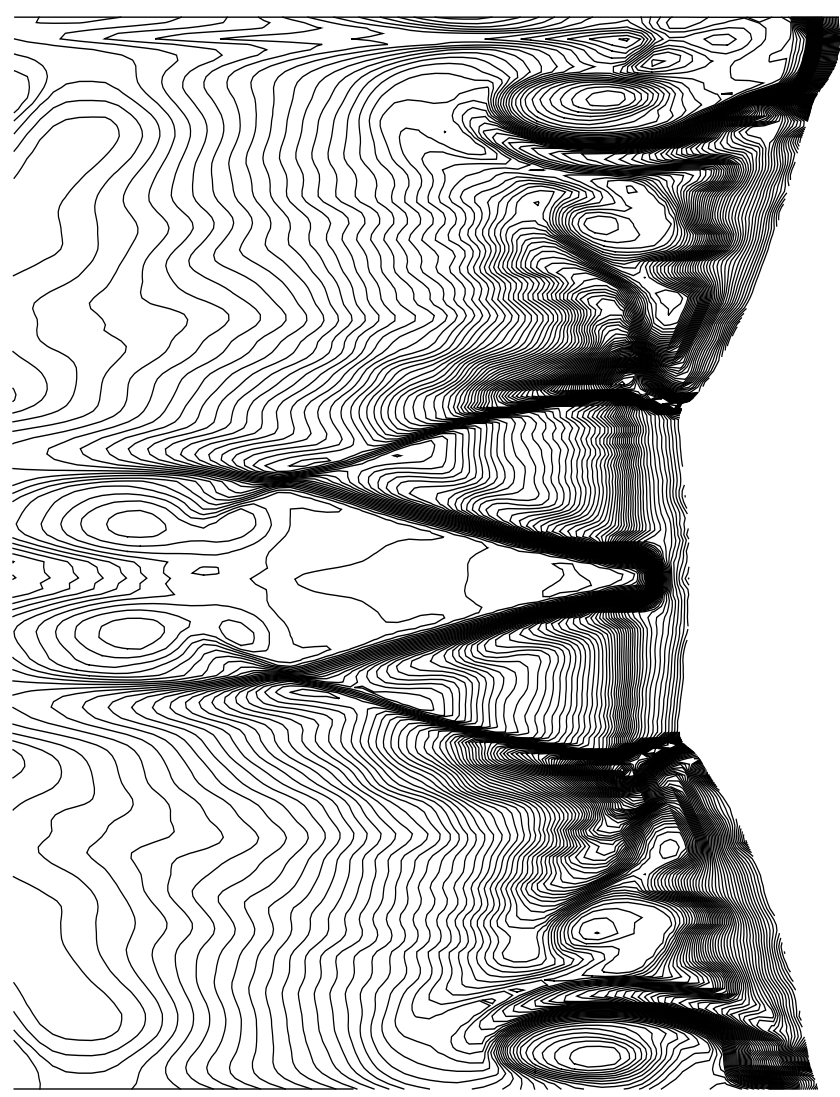

(b)

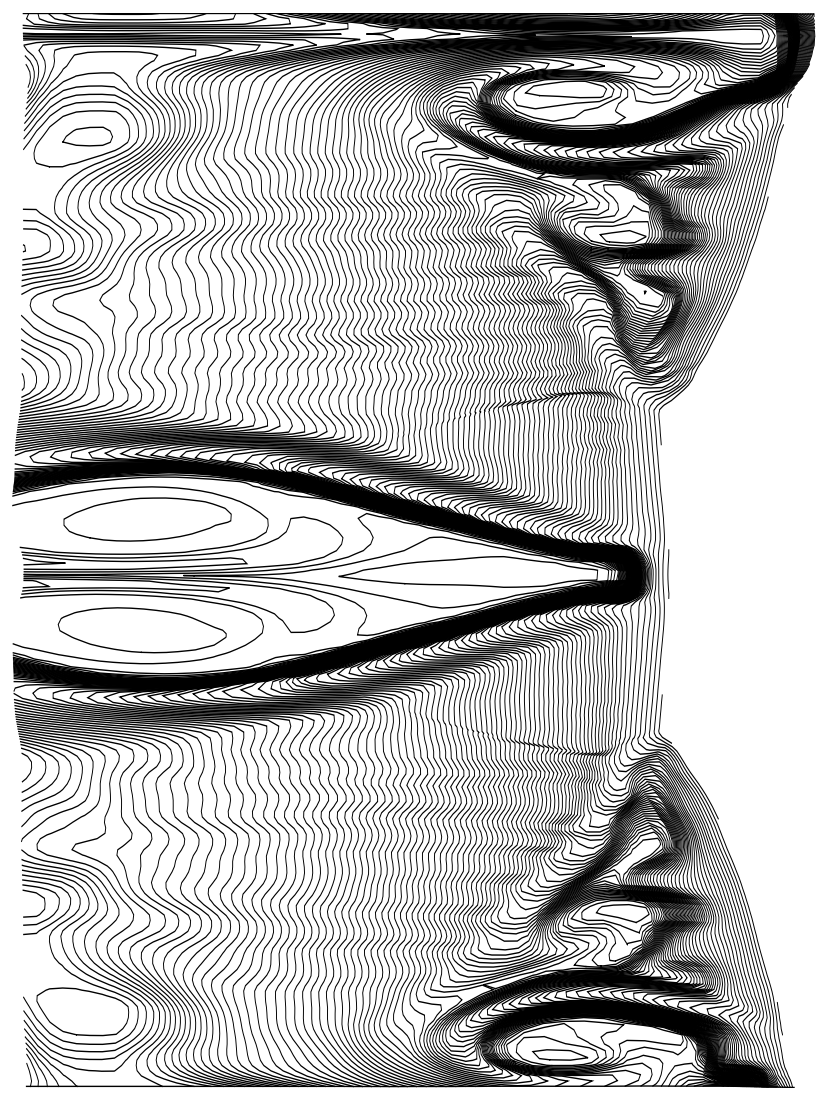

(d)

Figure 15. Density plots at $t=60.44$ for rectangular mesh (a), front tracking (b), front tracking with adaptation (c) (mesh in Fig.14a) calculation. Mass fraction for front tracking calculation (d). 

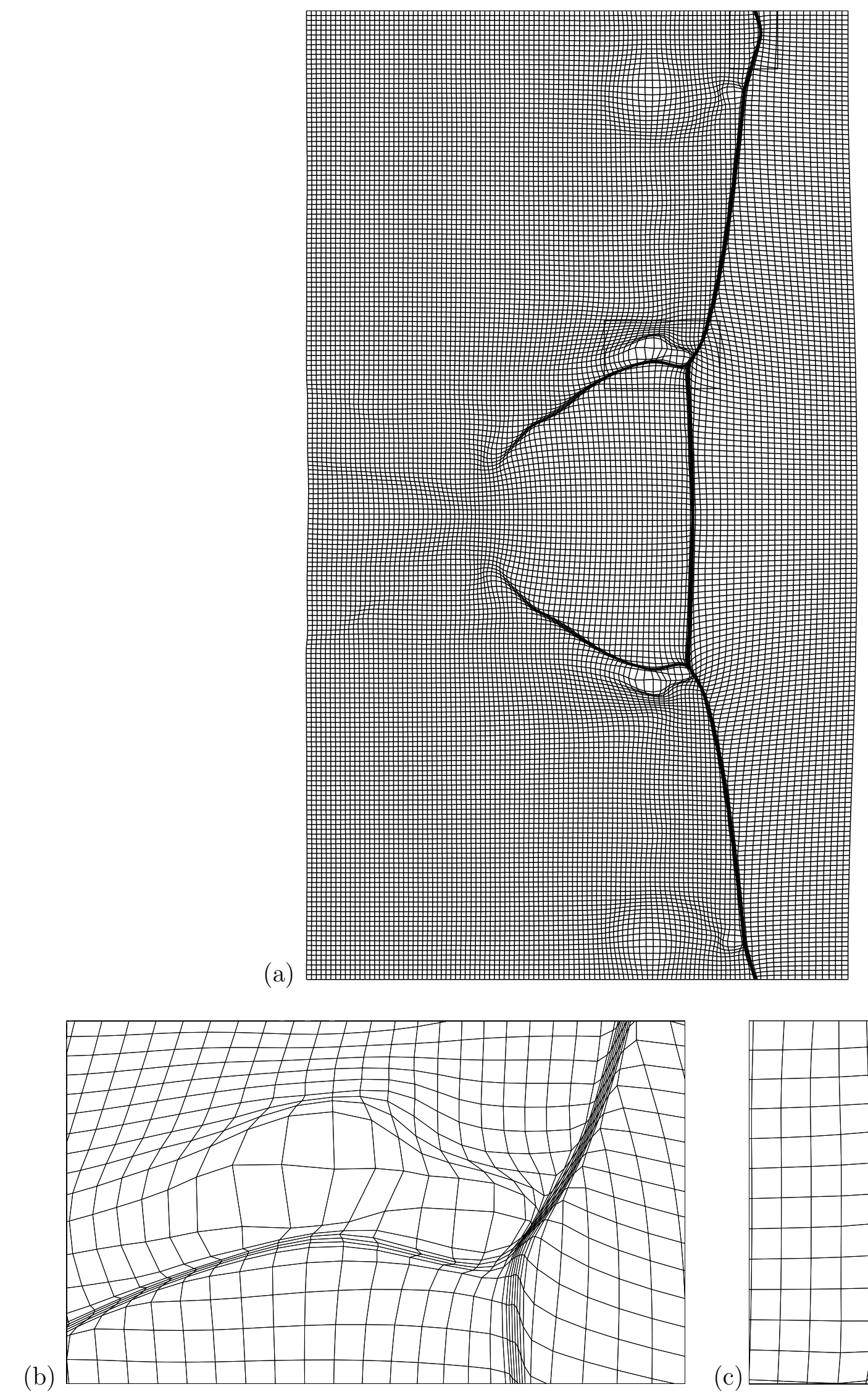

(c)

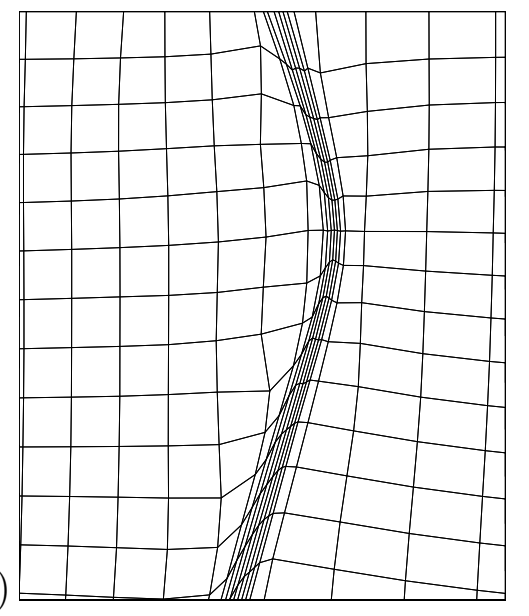

Figure 16. Global adaptation with $p$ as a control function $f$. Mesh (a) and close-up (b,c) at $t=60.44$; time step $\Delta t=10^{-10}-10^{-5} \cdot \Delta t_{\text {rect }}$. 


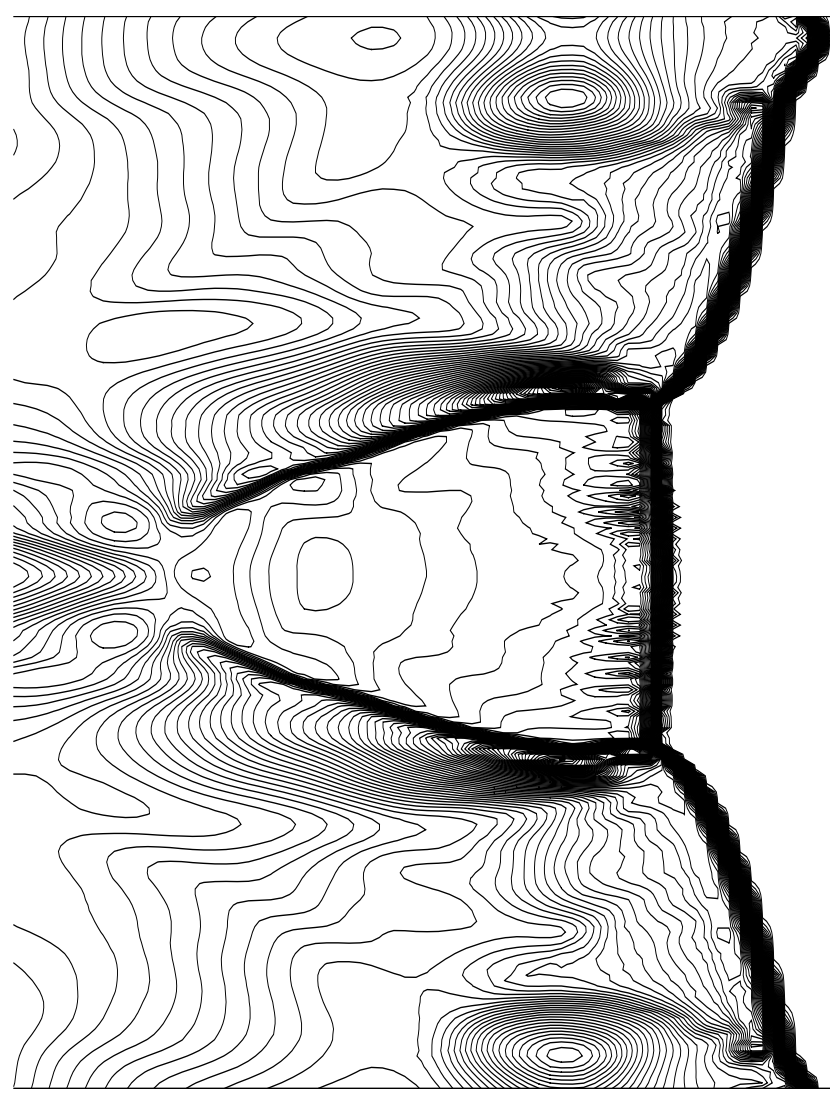

(a)

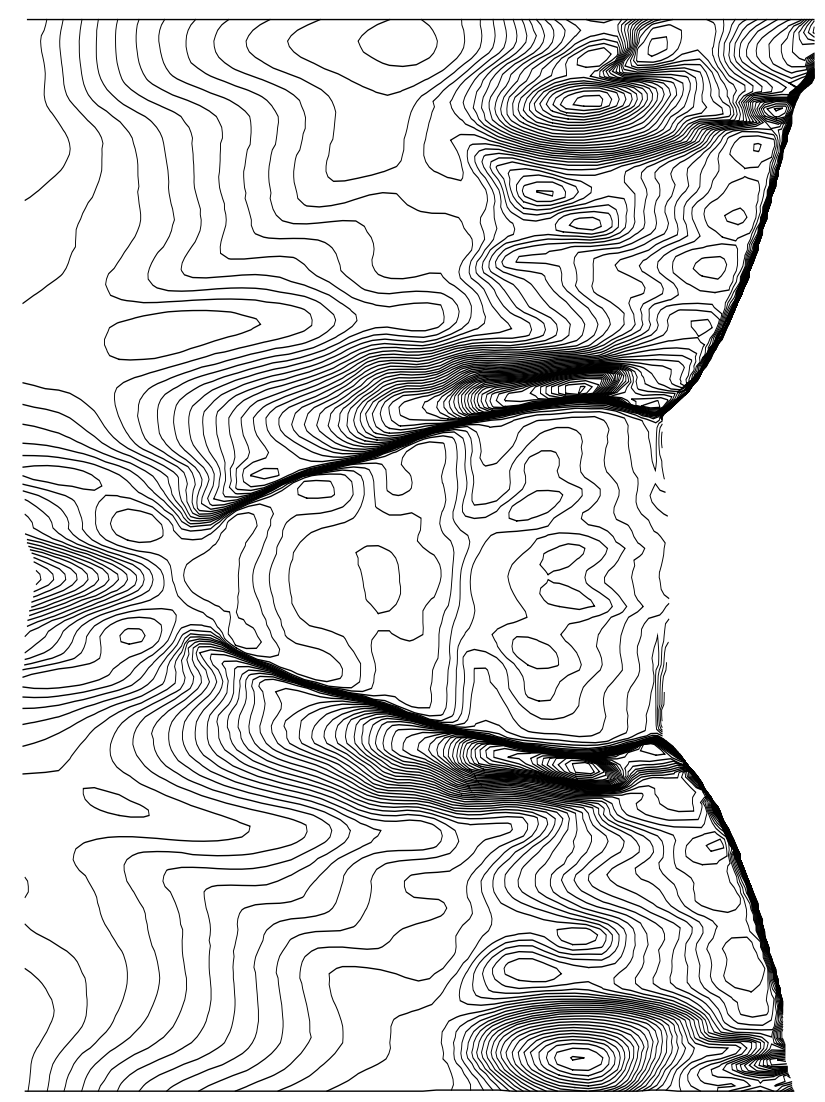

(c)

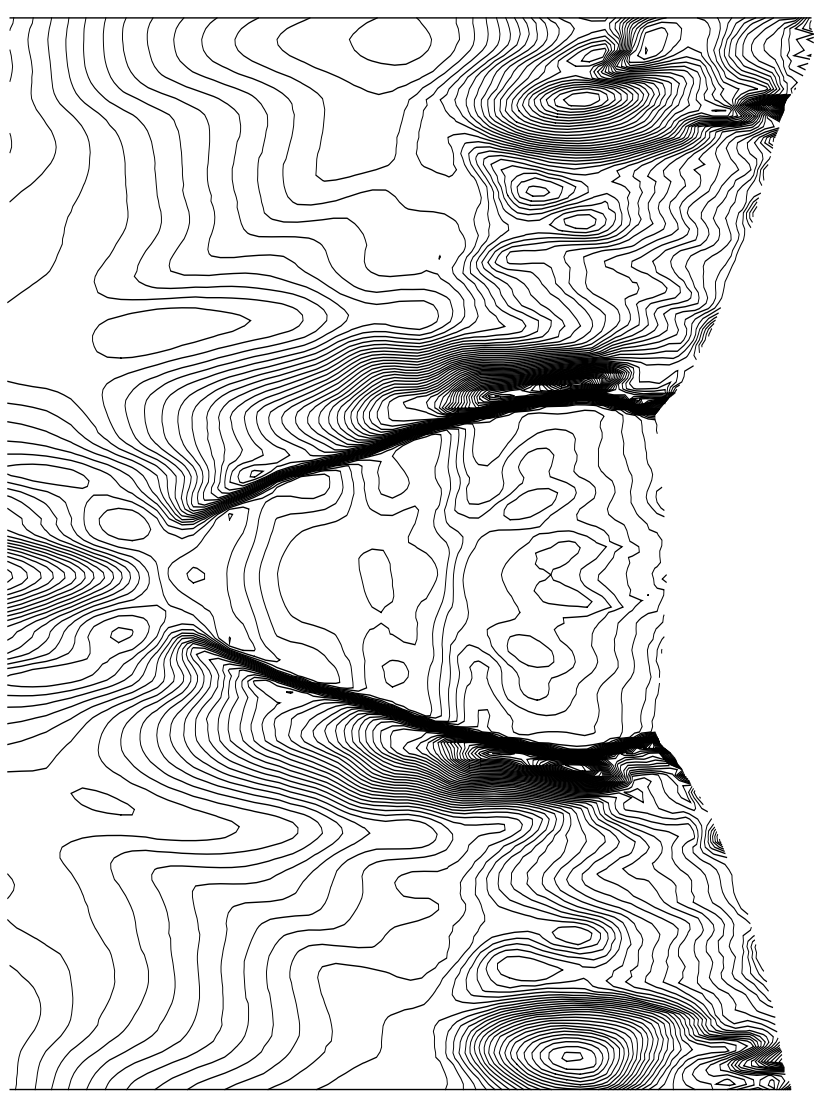

(b)

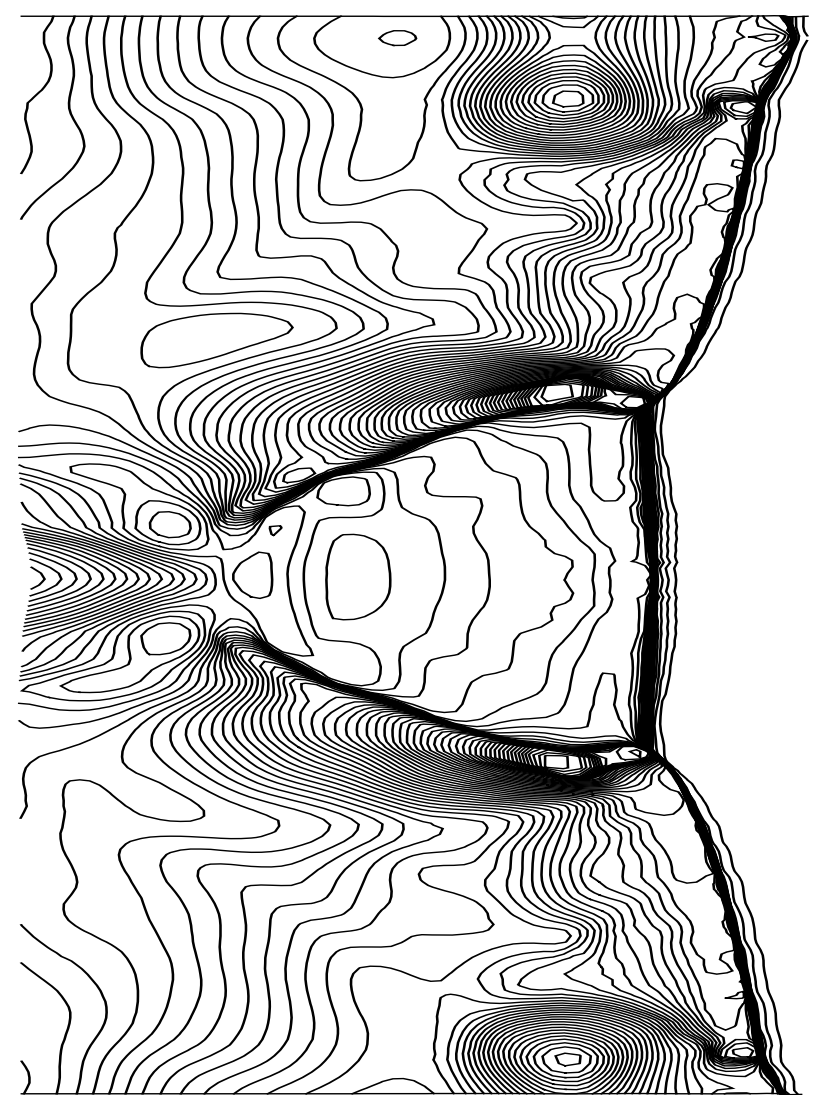

(d)

Figure 17. Pressure plots at $t=60.44$ for rectangular mesh (a), front tracking (b), front tracking with adaptation (c) (mesh in Fig.14b), and global adaptaion (d) (mesh in Fig.16) calculation. 


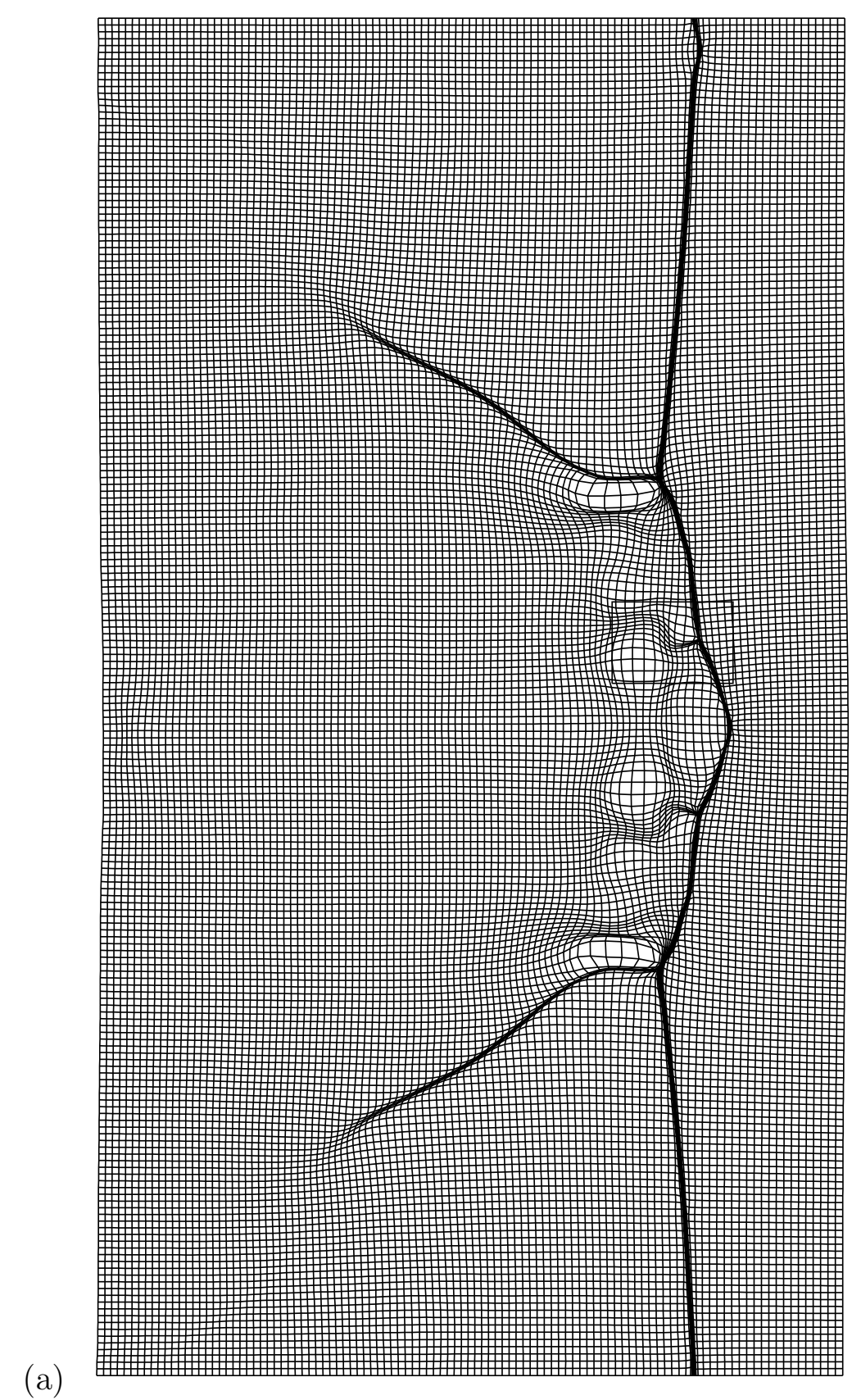

(a)

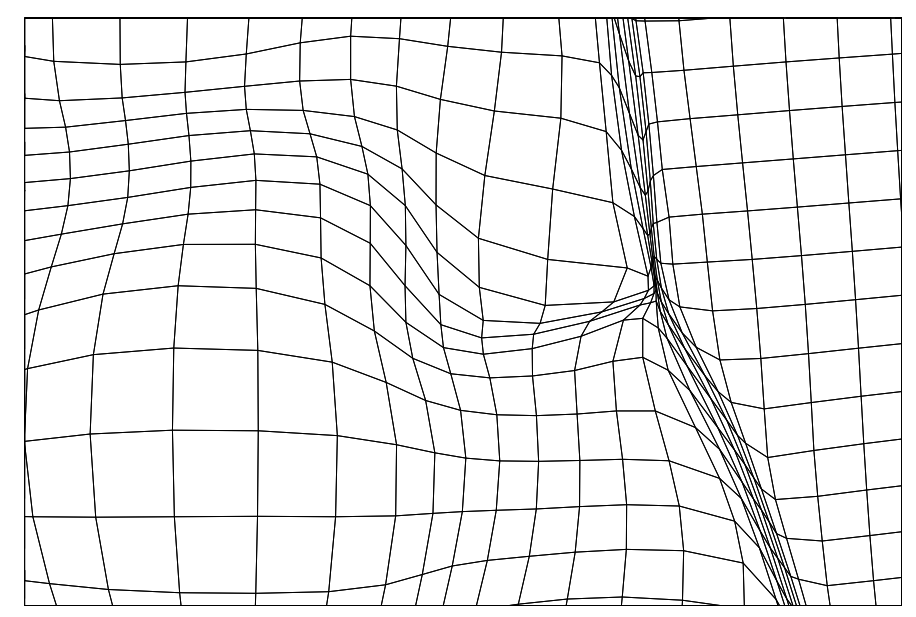

Figure 18. Global adaptation with $p$ as a control function $f$. Mesh (a) and close-up (b) at $t=61.59$. 


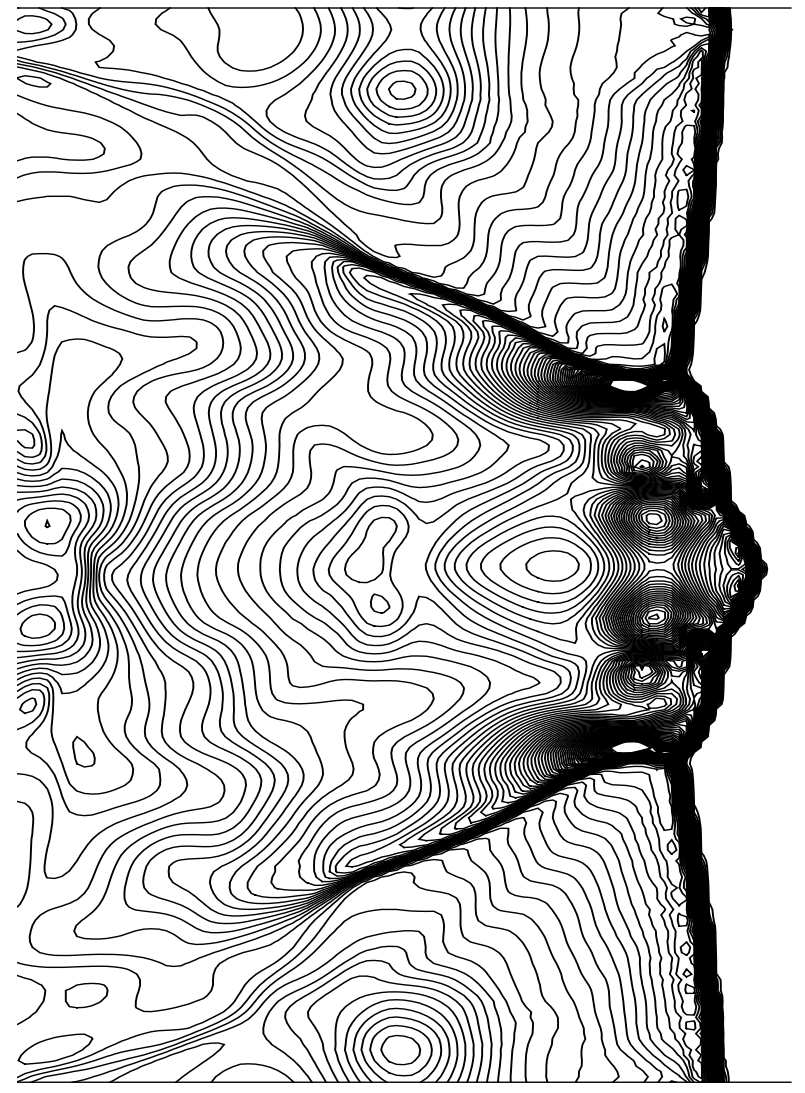

(a)

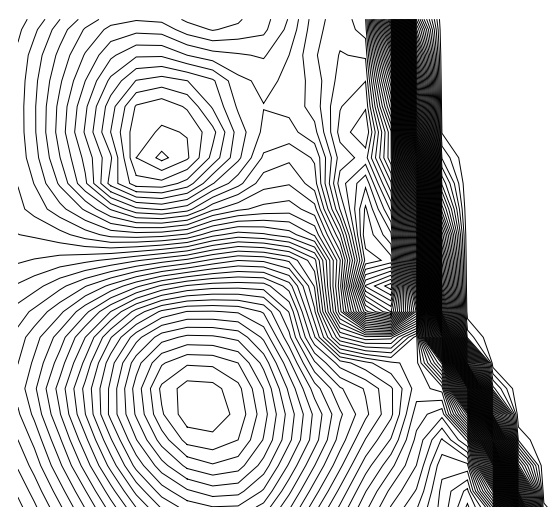

(c)

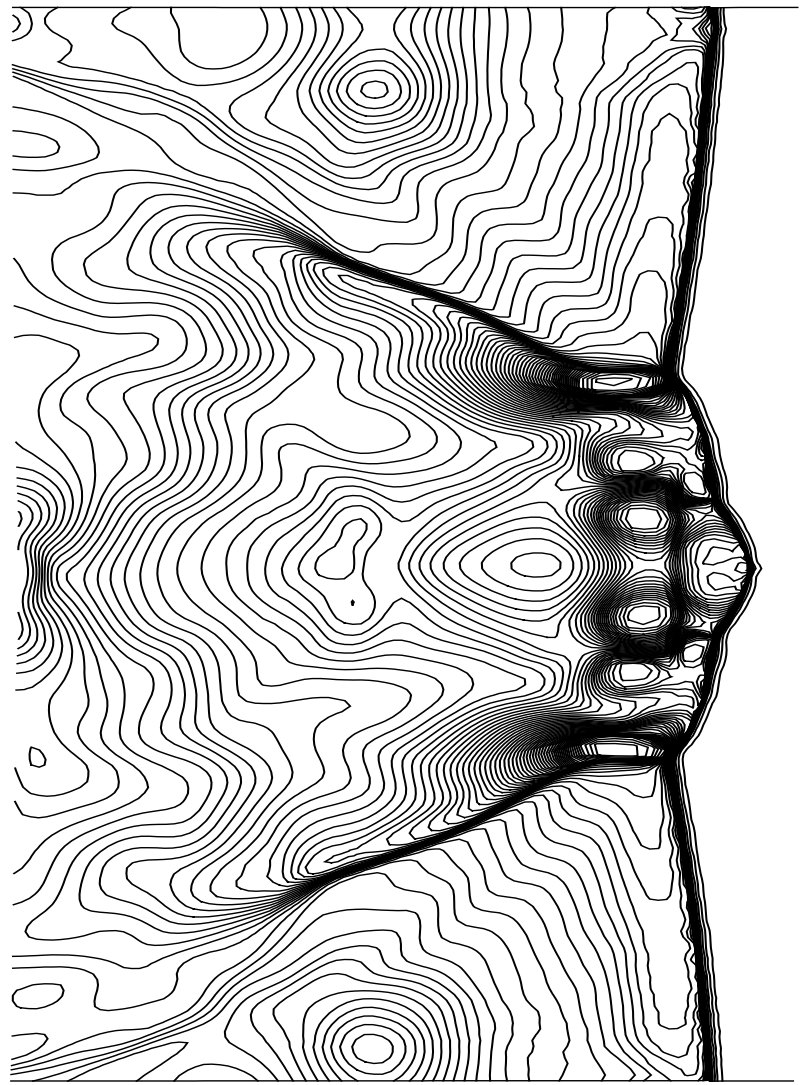

(b)

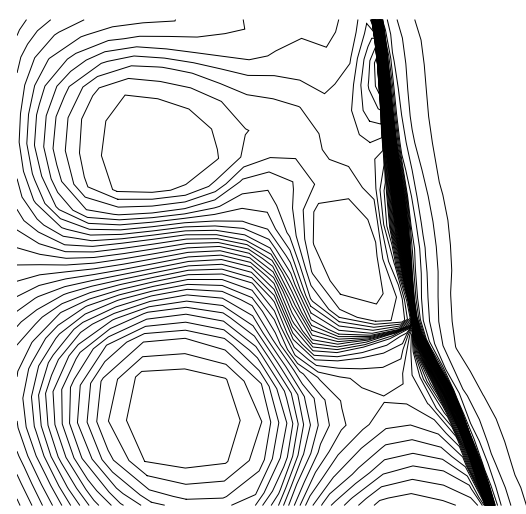

(d)

Figure 19. Pressure plots at $t=61.59$ computed on the rectangular (a) and adapted (b) mesh. Close-up for the rectangular (c) and adapted (d) mesh (domain, depicted in Fig 18b). 